University of Nebraska - Lincoln

DigitalCommons@University of Nebraska - Lincoln

USDA Forest Service / UNL Faculty Publications U.S. Department of Agriculture: Forest Service -National Agroforestry Center

2010

Effects of chronic ammonium sulfate treatment on the forest at the Bear Brook Watershed in Maine

Jose Alexander Elvir

Escuela Nacional de Ciencias Forestales, alexit98@yahoo.com

G. Bruce Wiersma

University of Maine

Suzanne Bethers

USDA Forest Service

Peter Kenlan

University of Maine

Follow this and additional works at: https://digitalcommons.unl.edu/usdafsfacpub

Elvir, Jose Alexander; Wiersma, G. Bruce; Bethers, Suzanne; and Kenlan, Peter, "Effects of chronic ammonium sulfate treatment on the forest at the Bear Brook Watershed in Maine" (2010). USDA Forest Service / UNL Faculty Publications. 248.

https://digitalcommons.unl.edu/usdafsfacpub/248

This Article is brought to you for free and open access by the U.S. Department of Agriculture: Forest Service -National Agroforestry Center at DigitalCommons@University of Nebraska - Lincoln. It has been accepted for inclusion in USDA Forest Service / UNL Faculty Publications by an authorized administrator of DigitalCommons@University of Nebraska - Lincoln. 


\title{
Effects of chronic ammonium sulfate treatment on the forest at the Bear Brook Watershed in Maine
}

\author{
Jose Alexander Elvir • G. Bruce Wiersma • \\ Suzanne Bethers · Peter Kenlan
}

This article is a U.S. government work, and is not subject to copyright in the United States.

Received: 14 July 2009 / Accepted: 9 April 2010 / Published online: 17 June 2010

(C) Springer Science+Business Media B.V. 2010

\begin{abstract}
At the Bear Brook Watershed in Maine (BBWM), the forest tree composition was characterized and the effects of the chronic ammonium sulfate $\left(\left(\mathrm{NH}_{4}\right)_{2} \mathrm{SO}_{4}\right)$ treatment on basal area growth, foliar chemistry, and gas exchange were investigated on forest species. The BBWM is a paired watershed forest ecosystem study with one watershed, West Bear (WB), treated since 1989 with $26.6 \mathrm{~kg} \mathrm{~N} \mathrm{ha}^{-1}$ year $^{-1}$ and $30 \mathrm{~kg} \mathrm{~S}^{-1}$ year ${ }^{-1}$ applied bimonthly as $\left(\mathrm{NH}_{4}\right)_{2} \mathrm{SO}_{4}$, while the other watershed, East Bear (EB), serves as a reference. Tree species richness, density, and mortality were found to be similar between watersheds. Basal area increment was estimated from red spruce and sugar maple, showing that, for the first
\end{abstract}

J. A. Elvir $(\bowtie)$

Escuela Nacional de Ciencias Forestales,

Apt. Postal \#2, Siguatepeque, Honduras

e-mail: alexit98@yahoo.com

G. B. Wiersma · P. Kenlan

CRSF, University of Maine, 263 Nutting Hall,

Orono, ME, USA

S. Bethers

Forest Health Management, Rocky Mountain Region, USDA Forest Service, 216 North Colorado Street, Gunnison, CO 81230, USA

P. Kenlan

Maine Island Trail Association, 58 Fore Street, \#30-3, Portland, ME 04101, USA
7 years of treatment, it was significantly higher for sugar maple growing in WB compared to EB, but no differences were observed for red spruce between watersheds. However, the initial higher sugar maple basal area growth in WB subsequently decreased after 8 years of treatment. Foliar chemical analysis performed in trees, saplings, and ground flora showed higher $\mathrm{N}$ concentrations in the treated WB compared to the reference EB. But, foliar cation concentrations, especially $\mathrm{Ca}$ and $\mathrm{Mg}$, were significantly lower for most of the species growing in WB compared with those growing in EB. For sugar maple, foliar $\mathrm{N}$ was higher on $\mathrm{WB}$, but there were no differences in foliar $\mathrm{Ca}$ and $\mathrm{Mg}$ concentrations between treated and reference watersheds. In addition, only sugar maple trees in the treated WB showed significantly higher photosynthetic rates compared to reference EB trees.

Keywords Species richness • Forest structure • Acidic deposition - Basal area growth • Foliar nutrients - Gas exchange

\section{Introduction}

Elevated nitrogen (N) and sulfur (S) deposition effects on forest growth remain a focus of interest because of incomplete understanding of how forest growth responds to $\mathrm{N}$ - and S-induced 
changes in nutrient availability. Atmospheric $\mathrm{N}$ and $\mathrm{S}$ deposition to forest ecosystems increased during the twentieth century due to human activities (Aber et al. 1991; Dise and Wright 1995; Reynolds et al. 1998; Tietema and Beier 1995). Recently, atmospheric $\mathrm{N}$ deposition has stabilized or increased slightly in the US while S deposition has decreased in most regions (Fenn et al. 2003; Lehmann et al. 2005; NADP 2002-2006).

Elevated atmospheric $\mathrm{N}$ deposition results in increased soil $\mathrm{N}$ availability, and in $\mathrm{N}$-limited ecosystems, increased plant $\mathrm{N}$ uptake (Aber et al. 1989, 1998; Driscoll et al. 2001; Weber and Wiersma 1997; White et al. 1999), yielding higher foliar N concentrations (Bertills and Näsholm 2000; Elvir et al. 2005, 2006; Magill et al. 1997) and increased forest growth (Elvir et al. 2003; Lloyd 1999; Magill et al. 1997; Spiecker et al. 1996). However, increased $\mathrm{N}$ deposition may have indirect negative impacts on forest growth (Bertills and Näsholm 2000; Oren et al. 1993). For example, forests under elevated $\mathrm{N}$ and $\mathrm{S}$ deposition might experience nutrient imbalances and growth decline due to changes in soil chemistry (Aber et al. 1989; Buchmann et al. 1995; DeHayes et al. 1999; Johnson et al. 1994; Oren et al. 1993; Vitousek et al. 1997). As $\mathrm{N}$ accumulates in the soil, ecosystem $\mathrm{N}$ retention capacity can be surpassed, resulting in increased leaching of $\mathrm{NO}_{3}^{-}$and base cations (Norton et al. 1999), and subsequently, reducing their availability for plant uptake. Also, as soil acidifies during base cation depletion, aluminum ( $\mathrm{Al}$ ) is mobilized and may be absorbed at toxic levels by passive root uptake (McNulty et al. 1996; Shortle and Smith 1988). Aluminum can also displace base cations from the soil rooting zone, reducing the uptake of major nutrients, furthering plant nutrient deficiencies (Cronan 1989).

The long-term ammonium sulfate $\left(\left(\mathrm{NH}_{4}\right)_{2} \mathrm{SO}_{4}\right)$ addition experiment at the Bear Brook Watershed in Maine (BBWM) provides a unique opportunity to study changes in forests due to enhanced $\mathrm{N}$ and $\mathrm{S}$ deposition. The BBWM has two contiguous watersheds, West Bear (WB) and East Bear (EB), which initially were similar in chemistry, biology, physical attributes, and hydrology (Norton et al. 1999). Ambient deposition at the beginning of the experiment (1989) was $8.4 \mathrm{~kg} \mathrm{ha}^{-1}$ year $^{-1}$ for $\mathrm{N}$ and $14.4 \mathrm{~kg} \mathrm{ha}^{-1}$ year $^{-1}$ for $\mathrm{S}$ (Kahl et al.
1999; Norton et al. 1999; Wang and Fernandez 1999). The $\mathrm{N}+\mathrm{S}$ addition treatment persists on bimonthly $\left(\mathrm{NH}_{4}\right)_{2} \mathrm{SO}_{4}$ amendments to WB since 1989 of $26.6 \mathrm{~kg} \mathrm{~N}^{-1}$ year $^{-1}$ and $30 \mathrm{~kg} \mathrm{~S} \mathrm{ha}^{-1}$ year $^{-1}$ (Norton et al. 1999).

Hydrologic and chemical monitoring during two pretreatment years and the first 6 years of treatment (1987-1995) indicated that $\left(\left(\mathrm{NH}_{4}\right)_{2} \mathrm{SO}_{4}\right)$ addition produced significant changes in stream water chemistry with elevated export of base cations, lower $\mathrm{pH}$ and alkalinity, and increased dissolved aluminum (Al), $\mathrm{SO}_{4}$, and $\mathrm{NO}_{3}^{-}$(Norton et al. 1999; Uddameri et al. 1995). After 7 years of treatment, export of base cations declined and dissolved and particulate $\mathrm{Al}$, phosphorous $(\mathrm{P})$, and iron $(\mathrm{Fe})$ increased. Retention of added $\mathrm{N}$ in $\mathrm{WB}$ has been $\sim 80 \%$ (Jefts et al. 2004; Kahl et al. 1999). Fernandez et al. $(1999,2003)$ indicated that the treatment induced the depletion of base cations $(\mathrm{Ca}, \mathrm{Mg}$, and $\mathrm{K}$ ) from soil exchangeable pools in the WB watershed. The treated WB watershed developed higher rates of net $\mathrm{N}$ mineralization and net nitrification, consistent with the symptoms of $\mathrm{N}$ saturation (Jefts et al. 2004).

This study is part of the ongoing long-term research evaluating the effects of increased $\mathrm{N}$ and $\mathrm{S}$ deposition on the geochemistry and biology of the treated watershed. This chapter reviews studies addressing the effects of the treatment on the forest vegetation, including growth, mortality, and foliar chemistry, and provides new data after 18 years of treatment of the WB watershed. This chapter summarizes and discusses the results for the overstory (Elvir et al. 2003, 2005, 2006), ground flora (Kenlan 2006), and sugar maple sapling physiology (Bethers 2008; Bethers et al. 2009).

\section{Study site}

The BBWM site is in eastern Maine, USA ( $44^{\circ} 52^{\prime} 15^{\prime \prime}$ latitude, $68^{\circ} 06^{\prime} 25^{\prime \prime}$ longitude) and includes two contiguous forested watersheds, West Bear (WB; 10.77 ha) and East Bear (EB; 11.42 ha). The hydrology, soils, vegetation, topography, relief, aspect, and exposure are similar 
Table 1 Proportion of each forest cover type for the two watersheds, West Bear Brook (WB) with $\left(\left(\mathrm{NH}_{4}\right)_{2} \mathrm{SO}_{4}\right)$ addition treatment and East Bear Brook (EB) an adjacent reference, within the BBWM

\begin{tabular}{|c|c|c|c|c|}
\hline \multirow[t]{2}{*}{ Cover type } & \multicolumn{2}{|c|}{ WB (treated) } & \multicolumn{2}{|c|}{ EB (reference) } \\
\hline & Hectares & Percent & Hectares & Percent \\
\hline $\begin{array}{l}\text { Hardwood } \\
(<21 \% \\
\text { softwood })\end{array}$ & 3.56 & 33.07 & 3.99 & 34.98 \\
\hline $\begin{array}{c}\text { Mixed wood } \\
(21-65 \% \\
\text { softwood) }\end{array}$ & 3.87 & 35.90 & 5.13 & 45.04 \\
\hline $\begin{array}{l}\text { Softwood } \\
\qquad>65 \% \\
\text { softwood) }\end{array}$ & 3.34 & 31.03 & 2.28 & 19.98 \\
\hline Total & 10.77 & 100.00 & 11.40 & 100.00 \\
\hline
\end{tabular}

From Wang (1993)

in both watersheds (Uddameri et al. 1995). Red spruce (Picea rubens Sarg.), American beech ( $\mathrm{Fa}$ gus grandifolia Ehrh.), sugar maple (Acer saccharum Marsh.), red maple (Acer rubrum L.), and yellow birch (Betula alleghaniensis Britt.) comprise up to $98 \%$ of the live trees distributed into three cover types: hardwood, softwood, and mixed wood. These cover types occur in both watersheds (Table 1). The hardwood and mixed wood stands are secondary forests since they underwent a major logging around 1945 (Champion International Corporation records cited in Norton et al. 1999). The softwood is mostly even-aged red spruce $>100$ years old (Elvir et al. 2003), which occurs primarily in the upper third of each watershed.

\section{Methods}

Forest monitoring plots

In 2000, 100 permanent vegetation plots were established at the BBWM. Their location was based on an existing south-north east-west grid system with benchmarks located every $30 \mathrm{~m}$ (Fig. 1). Using the grid, 50 grid points were randomly selected in each watershed, proportionally distributed per area of each cover type (33 plots hardwood, 36 plots mixed wood, and 31 plots softwood). A 15-m by $15-\mathrm{m}$ plot $\left(225 \mathrm{~m}^{2}\right)$ was established with each grid point at the plot center.

The diameter of all trees $\geq 12 \mathrm{~cm}$ was measured at diameter at breast height (DBH, $1.3 \mathrm{~m}$ above the ground line on the uphill side of the tree) within each plot in 2000. A mark was painted at $1.3 \mathrm{~m}$ to maintain the $\mathrm{DBH}$ measure at the same height in consecutive years. Trees were marked with the plot identification and a consecutive number on an aluminum tag nailed to the stem. The plots were revisited and trees were remeasured in 2002, 2003, 2006, and 2008. At each new measurement, mortality and ingrowth were recorded. For each year, we calculated mean species DBH, density, mortality, and ingrowth per hectare.

\section{Basal area increment growth}

In 2000, basal area increment (BAI) was estimated by extracting cores from red spruce and sugar maple on both the treated WB and the reference EB watersheds (Elvir et al. 2003). Within each watershed, sample trees were restricted to dominants or codominants with a single stem and with an overall healthy appearance to reduce variability associated with factors other than the treatment. From the subset of suitable trees, 25 sugar maple and 25 red spruce trees were randomly selected. A 4.30-mm diameter increment borer was used to extract two cores from opposite sides of each selected tree on the cross slope at a height of approximately $1.3 \mathrm{~m}$ above the ground (Andrews and Siccama 1995; Bondietti et al. 1990; Peterson et al. 1990).

Individual annual ring widths for tree cores were counted and measured using an Olympus ${ }^{\mathrm{TM}}$ SZ-STB1 microscope and a Velmex measuring stage connected to a computer. A Measure J2X Tree Ring Measuring program was used. Crossdating was done for the last 26 years following the methodology of Glock (1937). Cores were discarded if the annual rings were indistinct or if temporal patterns of ring width did not show reasonable agreement between cores. Consequently, cores from eight sugar maple trees (three trees from the treated $\mathrm{WB}$ and five trees from the reference EB) were discarded. No red spruce cores 


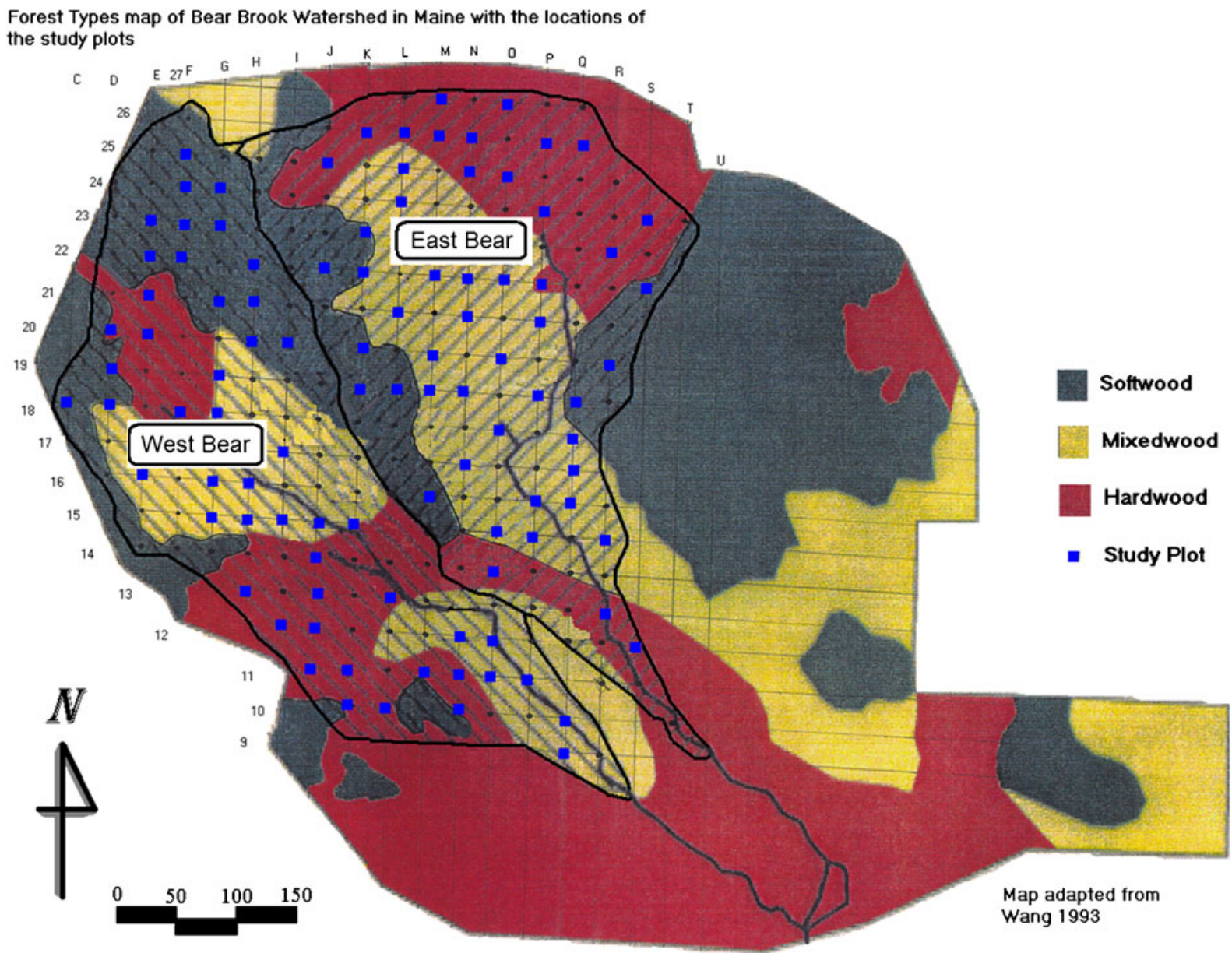

Fig. 1 The BBWM, cover types, grid system, and permanent plots (small squares). West Bear Brook (WB) with $\left(\left(\mathrm{NH}_{4}\right)_{2} \mathrm{SO}_{4}\right)$ addition treatment and East Bear Brook $(E B)$ the reference

were discarded. Ring widths for the two cores of the same tree were averaged by year. Cumulative BAI per year was calculated from the average of the two cores extracted from each tree. Mean yearly BAI from 1980 to 1999 for each species was compared between watersheds using a oneway analysis of variance (ANOVA; SAS 1999). ANOVA assumptions were tested using the UNIVARIATE Procedure (SAS 1999) for normal distribution of residuals and Levine's test for equality of variances.

In 2006, with 16 years of $\mathrm{N}+\mathrm{S}$ addition treatment and 6 years after the initial tree coring in 2000 , cores were extracted again from the same 25 trees per species in each watershed. The same methodology as in 2000 was applied.
Foliar tree chemistry

The three most dominant tree species at the BBWM (American beech, sugar maple, and red spruce) were sampled for foliar chemical analysis in 1993 (White et al. 1999), 1998-2000, and 20022003 (Elvir et al. 2005). In all years, dominant or codominant healthy trees of each species were randomly selected in each watershed. Details on those collections and laboratory analyses are in White et al. (1999) and Elvir et al. (2005). Number of trees sampled per species and time of sample collection varied among collection periods. In 1993, White et al. (1999) collected foliage samples from 36 American beech, 31 sugar maple, and 21 red spruce from the treated WB 
watershed and 23 American beech, 11 sugar maple, and 29 red spruce on the reference EB watershed during the first week of September 1993 for hardwood species and in mid-October for red spruce. In 1998-2000, Elvir et al. (2005) collected foliage samples from 20 trees of each species (American beech, sugar maple, and red spruce) in each watershed during the first 2 weeks of August for both hardwood and softwood species. In 2002-2003, Elvir et al. (2005) collected foliage samples from nine trees of each species in each watershed during the last week of July and first week of August for sugar maple, in mid-August for American beech, and during the first week of September for red spruce. Sampled trees were different in each time period (1993, 1998-2000, 2002-2003). In 2006, foliage samples were collected from the same nine trees per species sampled in 2002 and 2003, plus six more trees per species for a total of 15 trees per species in each watershed. Sample collection procedures followed Elvir et al. (2005). For all time periods, foliage samples were collected from the south side of the upper crown of selected trees. Unwashed foliage samples were oven dried to a constant mass at $70^{\circ} \mathrm{C}$. Chemical analyses were performed by the Analytical Laboratory of the Maine Agricultural and Forest Experiment Station at The University of Maine. Total $\mathrm{N}$ was determined by combustion analysis or the Dumas method (Simonne et al. 1997). Other nutrient concentrations were determined by inductively coupled plasma atomic emission spectroscopy (Thermo Jarrell Ash model 975 ICP-OES).

\section{Physiology}

Photosynthetic and transpiration rates were measured in sugar maple, American beech, and red spruce trees in the 2002 and 2003 growing seasons (end of July and August in both years). As described by Elvir et al. (2005), gas-exchange measurements were made (between 8:00 A.M. and noon) on days that were sunny and relatively cloud-free for nine trees per species randomly selected in each watershed. Net photosynthetic and transpiration rates were measured with a LI-6400 Photosynthesis System (LI-COR 1998), equipped with a standard $6-\mathrm{cm}^{2}$ leaf cuvette (set for air flow at $500 \mathrm{mmol} \mathrm{s}^{-1}$ and leaf temperature at $25^{\circ} \mathrm{C}$ ) and a model 6400-02B light source (set at 2,000 mmol m${ }^{-2} \mathrm{~s}^{-1}$; LI-COR 1998). Measurements were made on fully expanded current-year needles of red spruce shoots and individual leaves of American beech and sugar maple from six randomly selected branches excised from the south side and upper crown. Leaves and current-year needles were also collected and pooled from the six selected branches per tree for nutrient chemical analysis. Samples were dried and analyzed by the same methods used for foliage chemistry.

\section{Ground flora}

In 2005, 101 plots, 53 located in EB and 48 in WB, were randomly selected using the grid intersections and proportionally distributed within each of three forest cover type. Each plot consisted of a $2-\mathrm{m}$ by $2-\mathrm{m}$ square divided into four $1-\mathrm{m}^{2}$ subplots (Fig. 2; Kenlan 2006). All plants shorter than $0.6 \mathrm{~m}$ in the northwest and southeast subplots were identified (for abundance and richness) and cover was estimated to the nearest $5 \%$. In the northeastern subplot, all plants shorter than

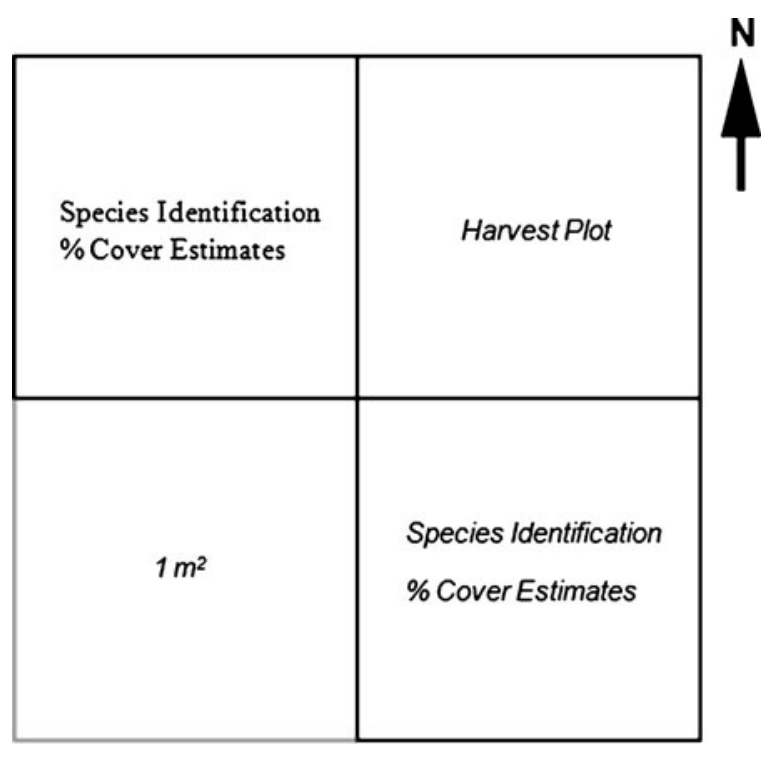

Fig. 2 Plot design for the ground flora study at the BBWM. West Bear Brook $(W B)$ watershed with $\left(\left(\mathrm{NH}_{4}\right)_{2} \mathrm{SO}_{4}\right)$ addition treatment and East Bear Brook $(E B)$ the reference watershed (from Kenlan 2006) 
$0.6 \mathrm{~m}$ were harvested at ground level to calculate total aboveground biomass. Plots were measured between July 5, 2005 and September 1, 2005.

Two abundant species of ground flora were selected for chemical analysis-A. saccharum and Uvularia sessilifolia. Foliage samples were collected from individuals in 25 plots randomly selected from the 101 forest floor plots in each watershed. Forest floor foliage was collected, processed, and analyzed by the same methods as for tree foliage.

Sugar maple saplings

As described in Bethers et al. (2009), sugar maple saplings in the treated (WB) and reference (EB) watersheds were compared for growth, foliar chemistry, and photosynthetic capacity. Sugar maple saplings were defined as individuals between 2 and $4 \mathrm{~m}$ in height and between 0.5 and $2.5 \mathrm{~cm} \mathrm{DBH}$ (Bethers 2008). Sugar maple saplings were measured for basal diameter (at $10 \mathrm{~cm}$ above ground level) and canopy cover in 84 plots, $15 \mathrm{~m} \times 15 \mathrm{~m}\left(225 \mathrm{~m}^{2}\right)$, distributed across both watersheds. Foliage was collected in the second week of August 2006 from saplings that were growing in $50 \%$ to full sunlight from EB (36 saplings) and WB (37 saplings) for foliar chemical analyses. Unwashed foliage was analyzed by the Analytical Laboratory of the Maine Agricultural and Forest Experiment Station at the University of Maine as previously described.

Photosynthesis was measured at the leaf level in the upper crowns in a 3-week period in late
July and early August on 15 saplings per watershed that occurred in small gaps where canopy openness was between $12 \%$ and $25 \%$ (Bethers et al. 2009). Using a LI-6400 gas-exchange chamber system (LI-COR 1998), capacity was measured by varying $\mathrm{CO}_{2}$ concentrations in the chamber from ambient to near zero and ambient to complete saturation, producing what is known as an $A-C_{i}$ curve (Farquhar et al. 1980). $A-C_{i}$ curves provide estimates of photosynthetic parameters such as maximum carboxylation capacity $\left(\mathrm{Vc}_{\max }\right)$, maximum electron transport $\left(J_{\max }\right)$, and triose phosphate utilization (TPU). Within the leaf chamber, leaves were exposed to a saturating irradiance of $1,200 \mu \mathrm{mol} \mathrm{m} \mathrm{m}^{-2} \mathrm{~s}^{-1}$, VPD was kept above $-1.5 \mathrm{MPa}$, and leaf temperature at $20-26^{\circ} \mathrm{C}$. Eleven moist leaves per sapling were collected from the saplings and their leaf area was measured using an LI-3100 (LI-COR 1998); leaves were then dried and weighed to calculate leaf mass per area (LMA; in grams per square meter).

\section{Results}

Forest monitoring plots

Of the nine species found at BBWM, five (American beech, red spruce, sugar maple, red maple, and yellow birch) represented $\sim 98 \%$ and $\sim 97 \%$ of the total density in EB and WB, respectively. Other species included balsam fir (Abies balsamea (L.) Mill.), red oak (Quercus rubra L.), striped
Table 2 Density (live trees per hectare) of tree species from 2000 to 2008 at the BBWM: West Bear Brook (WB) watershed with $\left(\left(\mathrm{NH}_{4}\right)_{2} \mathrm{SO}_{4}\right)$ addition treatment and East Bear Brook (EB) the reference watershed

\begin{tabular}{|c|c|c|c|c|c|c|c|c|c|c|}
\hline \multirow[t]{3}{*}{ Species } & \multicolumn{10}{|c|}{ Year } \\
\hline & \multicolumn{2}{|c|}{2000} & \multicolumn{2}{|l|}{2002} & \multicolumn{2}{|l|}{2003} & \multicolumn{2}{|l|}{2006} & \multicolumn{2}{|l|}{2008} \\
\hline & $\overline{\mathrm{WB}}$ & EB & $\overline{\mathrm{WB}}$ & EB & $\mathrm{WB}$ & $\overline{E B}$ & $\mathrm{WB}$ & EB & $\overline{\mathrm{WB}}$ & $\mathrm{EB}$ \\
\hline American beech & 248 & 275 & 244 & 279 & 243 & 270 & 233 & 250 & 233 & 243 \\
\hline Sugar maple & 122 & 100 & 118 & 99 & 108 & 99 & 100 & 98 & 96 & 101 \\
\hline Red maple & 36 & 50 & 36 & 50 & 36 & 50 & 36 & 46 & 36 & 46 \\
\hline Yellow birch & 36 & 75 & 36 & 74 & 36 & 72 & 36 & 73 & 36 & 74 \\
\hline Striped maple & 15 & 6 & 13 & 4 & 8 & 4 & 5 & 2 & 4 & 2 \\
\hline Black cherry & 4 & 0 & 4 & 0 & 4 & 0 & 4 & 0 & 4 & 0 \\
\hline Red oak & 1 & 1 & 1 & 1 & 1 & 1 & 1 & 1 & 1 & 1 \\
\hline Red spruce & 329 & 224 & 321 & 219 & 316 & 219 & 313 & 208 & 307 & 196 \\
\hline Balsam fir & 0 & 5 & 0 & 3 & 0 & 3 & 0 & 2 & 0 & 2 \\
\hline Total & 791 & 736 & 773 & 729 & 752 & 718 & 728 & 680 & 717 & 665 \\
\hline
\end{tabular}


Table 3 Cumulative ingrowth (number of new trees per hectare) of tree species from 2000 to 2006 at the BBWM: West Bear Brook (WB) watershed with $\left(\left(\mathrm{NH}_{4}\right)_{2} \mathrm{SO}_{4}\right)$ addition treatment and East Bear Brook (EB) the reference watershed

\begin{tabular}{|c|c|c|c|c|c|c|c|c|c|c|}
\hline \multirow[t]{3}{*}{ Species } & \multicolumn{10}{|c|}{ Year } \\
\hline & \multicolumn{2}{|c|}{2000} & \multicolumn{2}{|l|}{2002} & \multicolumn{2}{|l|}{2003} & \multicolumn{2}{|l|}{2006} & \multicolumn{2}{|l|}{2008} \\
\hline & WB & $\mathrm{EB}$ & WB & $\mathrm{EB}$ & WB & $\mathrm{EB}$ & WB & $\mathrm{EB}$ & WB & $\mathrm{EB}$ \\
\hline American beech & 0 & 0 & 11 & 18 & 21 & 25 & 35 & 36 & 44 & 46 \\
\hline Sugar maple & 0 & 0 & 2 & 1 & 4 & 1 & 4 & 4 & 7 & 9 \\
\hline Red maple & 0 & 0 & 1 & 0 & 1 & 0 & 2 & 0 & 2 & 0 \\
\hline Yellow birch & 0 & 0 & 1 & 3 & 1 & 3 & 3 & 7 & 3 & 8 \\
\hline Striped maple & 0 & 0 & 0 & 0 & 0 & 0 & 0 & 0 & 0 & 0 \\
\hline Black cherry & 0 & 0 & 0 & 0 & 0 & 0 & 0 & 0 & 0 & 0 \\
\hline Red oak & 0 & 0 & 0 & 0 & 0 & 0 & 0 & 0 & 0 & 0 \\
\hline Red spruce & 0 & 0 & 1 & 1 & 2 & 3 & 5 & 4 & 8 & 6 \\
\hline Balsam fir & 0 & 0 & 0 & 0 & 0 & 0 & 0 & 0 & 0 & 0 \\
\hline Total & 0 & 0 & 16 & 23 & 29 & 32 & 49 & 51 & 64 & 69 \\
\hline
\end{tabular}

maple (Acer pensylvanicum L.), and black cherry (Prunus serotina Ehrh.; Table 2). Red spruce was the most abundant species (accounting for 37\% of the density in $\mathrm{EB}$ and $33 \%$ in $\mathrm{WB}$ ) followed by American beech and sugar maple (Table 2). American beech stands were infected with bark disease. The disease results when bark, attacked and altered by the beech scale, Cryptococcus fagisuga Lind., is invaded and killed by fungi, primarily Nectria coccinea (Houston et al. 1979).

Tree density (trees per hectare) decreased in both watersheds between 2000 and 2008 (Table 2). In 2000, density in EB and WB was 736 and 791 trees per hectare, respectively; by 2008, density was 665 and 717 trees per hectare in EB and WB, respectively. Mortality of trees in both watersheds between 2000 and 2008 was compensated in part by ingrowth (Table 3 ). Total species mortality for the 2000-2008 period was similar between watersheds with 140 and 139 dead trees per hectare in EB and WB, respectively (Table 4). American beech accounted for the highest number of dead trees, with 77 and 59 trees per hectare in EB and $\mathrm{WB}$, respectively, which might be attributed to its high abundance as well as an effect of the American beech bark disease. Total net mortality (live trees in 2000 minus live trees in 2008) was 71 and 75 dead trees per hectare representing $11 \%$ and $10 \%$ in EB and WB, respectively.

Diameter growth increased in both watersheds for all species (Table 5). In 2000, mean diameter was $20.8 \mathrm{~cm}$ in both watersheds; by 2008 , mean diameters were 22.1 and $22.6 \mathrm{~cm}$ representing $6 \%$ and $9 \%$ increase in $\mathrm{EB}$ and $\mathrm{WB}$, respectively. Although overall tree DBH growth increase was higher in WB, differences between watersheds were not significant. Average DBH growth was offset by tree mortality; for some species, a decline in average diameter was recorded in some years.
Table 4 Cumulative mortality (dead trees per hectare) of tree species from 2000 to 2008 at the BBWM: West Bear Brook (WB) watershed with $\left(\left(\mathrm{NH}_{4}\right)_{2} \mathrm{SO}_{4}\right)$ addition treatment and East Bear Brook (EB) the reference watershed

\begin{tabular}{|c|c|c|c|c|c|c|c|c|c|c|}
\hline \multirow[t]{3}{*}{ Species } & \multicolumn{10}{|c|}{ Year } \\
\hline & \multicolumn{2}{|c|}{2000} & \multicolumn{2}{|l|}{2002} & \multicolumn{2}{|l|}{2003} & \multicolumn{2}{|l|}{2006} & \multicolumn{2}{|l|}{2008} \\
\hline & WB & $\mathrm{EB}$ & WB & $\mathrm{EB}$ & WB & $\mathrm{EB}$ & WB & EB & WB & EB \\
\hline American beech & 0 & 0 & 14 & 13 & 26 & 29 & 48 & 55 & 59 & 77 \\
\hline Sugar maple & 0 & 0 & 5 & 3 & 17 & 3 & 26 & 7 & 33 & 8 \\
\hline Red maple & 0 & 0 & 0 & 0 & 1 & 0 & 1 & 4 & 2 & 4 \\
\hline Yellow birch & 0 & 0 & 1 & 3 & 1 & 5 & 1 & 9 & 2 & 9 \\
\hline Striped maple & 0 & 0 & 3 & 2 & 9 & 3 & 11 & 4 & 13 & 4 \\
\hline Black cherry & 0 & 0 & 0 & 0 & 0 & 0 & 0 & 0 & 0 & 0 \\
\hline Red oak & 0 & 0 & 0 & 0 & 0 & 0 & 0 & 0 & 0 & 0 \\
\hline Red spruce & 0 & 0 & 9 & 6 & 14 & 8 & 21 & 19 & 30 & 34 \\
\hline Balsam fir & 0 & 0 & 0 & 3 & 0 & 3 & 0 & 4 & 0 & 4 \\
\hline Total & 0 & 0 & 32 & 30 & 68 & 51 & 108 & 102 & 139 & 140 \\
\hline
\end{tabular}


Table 5 Mean DBH of tree species from 2000 to 2008 at the BBWM: West Bear Brook (WB) watershed with $\left(\left(\mathrm{NH}_{4}\right)_{2} \mathrm{SO}_{4}\right)$ addition treatment and East Bear Brook (EB) the reference watershed

\begin{tabular}{|c|c|c|c|c|c|c|c|c|c|c|}
\hline \multirow[t]{3}{*}{ Species } & \multicolumn{10}{|c|}{ Year } \\
\hline & \multicolumn{2}{|l|}{2000} & \multicolumn{2}{|l|}{2002} & \multicolumn{2}{|l|}{2003} & \multicolumn{2}{|l|}{2006} & \multicolumn{2}{|l|}{2008} \\
\hline & WB & EB & WB & EB & WB & EB & WB & EB & WB & EB \\
\hline American beech & 18.1 & 18.5 & 18.4 & 18.5 & 18.6 & 18.6 & 19.0 & 18.9 & 19.5 & 19.1 \\
\hline Sugar maple & 18.5 & 19.7 & 18.9 & 20.0 & 19.4 & 20.2 & 19.8 & 20.2 & 20.0 & 20.2 \\
\hline Red maple & 22.1 & 22.4 & 22.3 & 23.0 & 22.4 & 23.2 & 22.7 & 24.0 & 23.4 & 24.6 \\
\hline Yellow birch & 25.1 & 22.5 & 24.7 & 23.1 & 24.8 & 23.1 & 25.0 & 23.5 & 25.4 & 24.0 \\
\hline Striped maple & 15.1 & 14.7 & 14.9 & 14.8 & 14.7 & 14.4 & 14.5 & 14.6 & 14.3 & 15.4 \\
\hline Black cherry & 26.4 & 0 & 26.6 & 0 & 26.7 & 0 & 27.6 & 0 & 27.9 & 0 \\
\hline Red oak & 17.8 & 35.7 & 18.3 & 36.5 & 18.7 & 36.8 & 19.5 & 37.7 & 20.4 & 38.6 \\
\hline Red spruce & 23.2 & 23.3 & 23.8 & 23.8 & 24.1 & 24.0 & 24.7 & 24.9 & 25.3 & 25.6 \\
\hline Balsam fir & 0 & 16.5 & 0 & 16.3 & 0 & 16.4 & 0 & 16.1 & 0 & 16.6 \\
\hline Weighed mean & 20.8 & 20.8 & 21.2 & 21.1 & 21.5 & 21.2 & 22.1 & 21.8 & 22.6 & 22.1 \\
\hline
\end{tabular}

Basal area increment growth

The average annual BAI for red spruce for the 1980-1989 period was 10.13 and $9.33 \mathrm{~cm}^{2}$ year $^{-1}$ in EB and WB, respectively, while for the 19902005 period, it was $10.15 \mathrm{~cm}^{2}$ year $^{-1}$ for EB and $8.92 \mathrm{~cm}^{2}$ year $^{-1}$ for $\mathrm{WB}$, with no significant differences between watersheds. The red spruce annual BAI and trends were similar to those re - ported by Elvir et al. (2003). The mean annual BAI for the last 26 years followed the same trend in the reference EB and the treated WB watersheds (Fig. 3).

The average annual BAI for sugar maple for the 1980-1989 period was similar between watersheds with 9.45 and $9.59 \mathrm{~cm}^{2}$ year ${ }^{-1}$ in EB and WB, respectively, while for the 1990-2005 period, it was $7.61 \mathrm{~cm}^{2}$ year $^{-1}$ for EB and $10.26 \mathrm{~cm}^{2}$ year $^{-1}$

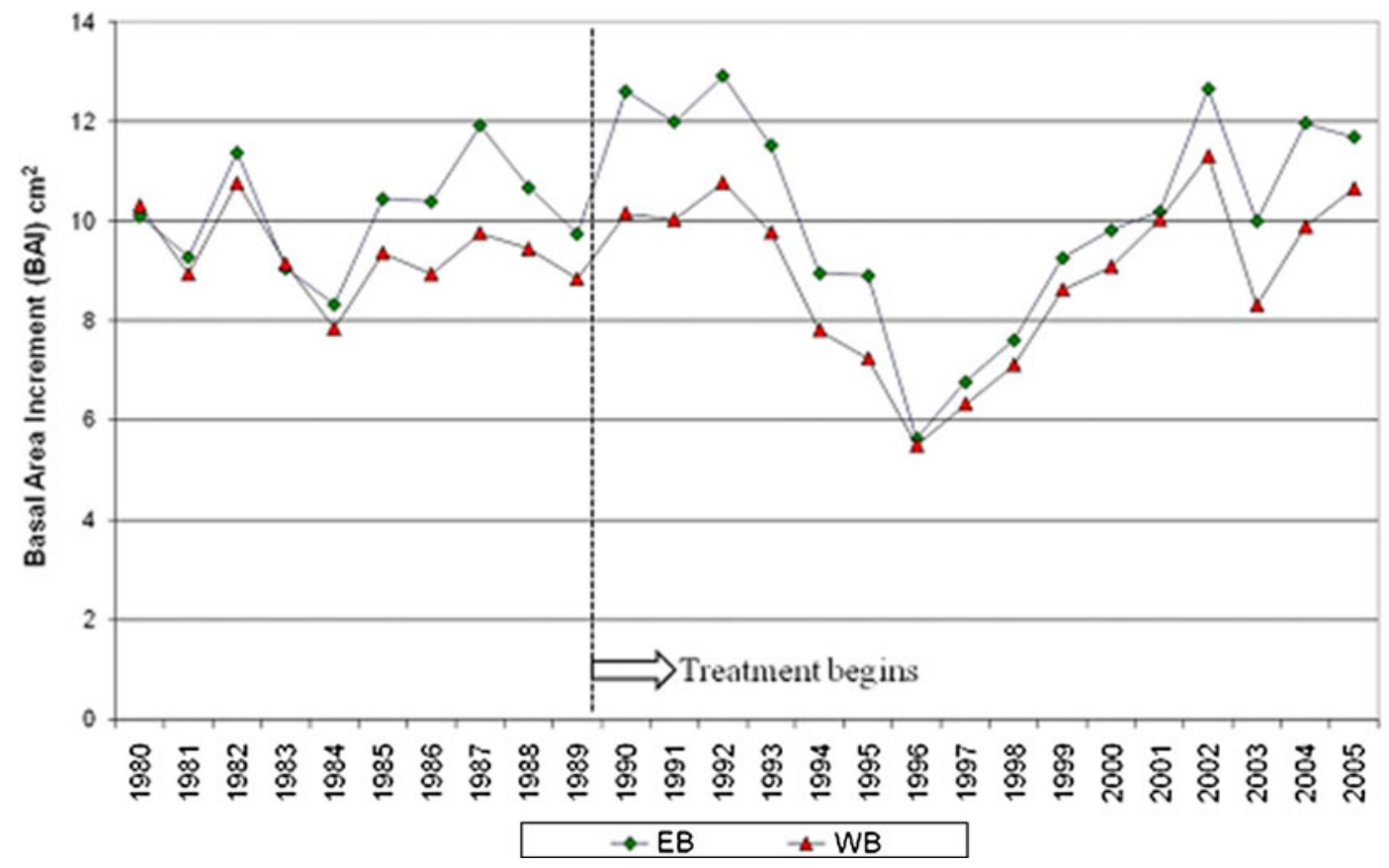

Fig. 3 Mean annual BAI for red spruce trees from the BBWM from 1980 to 2005 . West Bear Brook (WB) watershed with $\left(\left(\mathrm{NH}_{4}\right)_{2} \mathrm{SO}_{4}\right)$ addition treatment and East Bear Brook $(E B)$ the reference watershed 


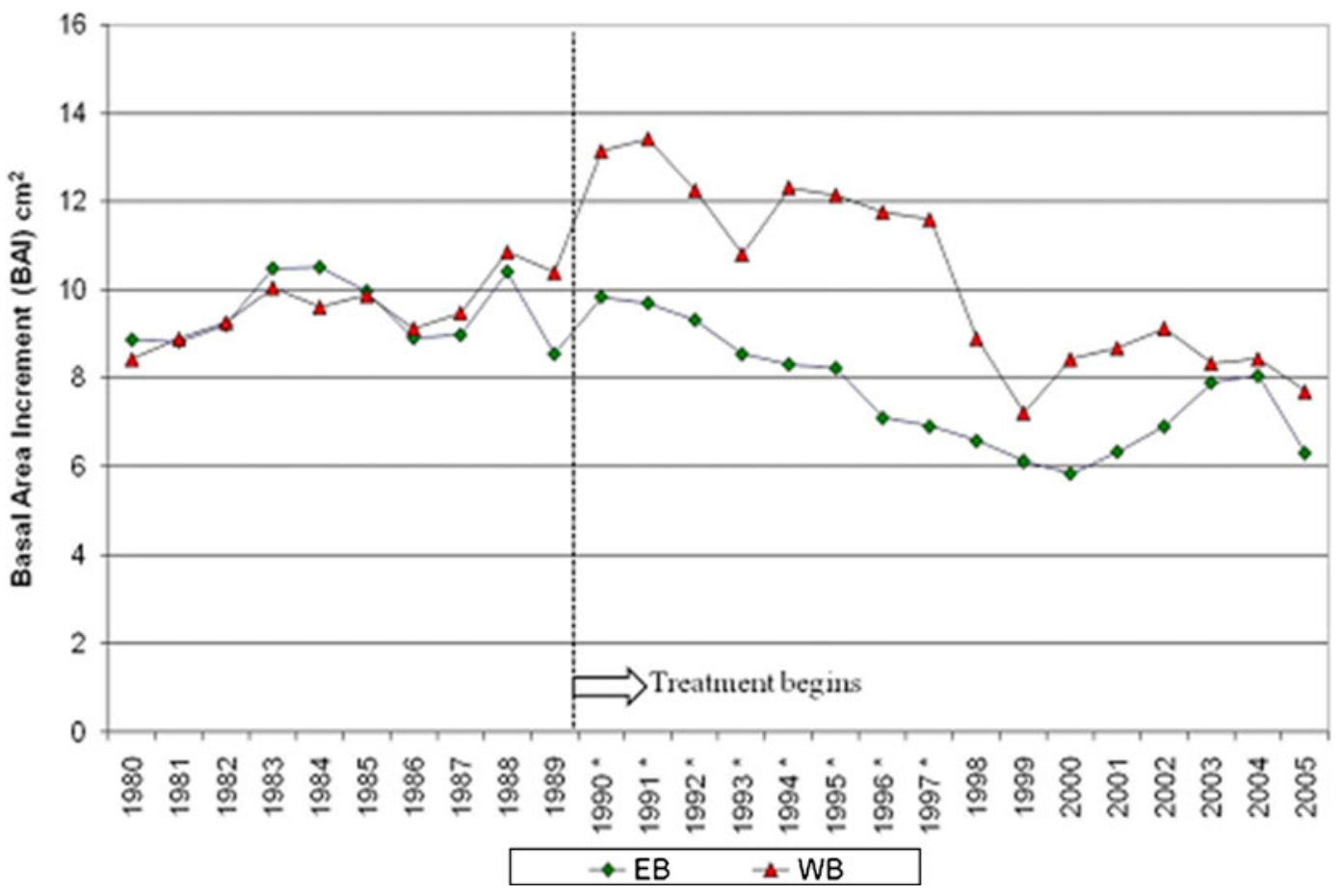

Fig. 4 Mean annual BAI for sugar maple trees from the BBWM from 1980 to 2005. West Bear Brook (WB) watershed with $\left(\left(\mathrm{NH}_{4}\right)_{2} \mathrm{SO}_{4}\right)$ addition treatment and East Bear
Brook $(E B)$ the reference watershed. The asterisk indicates significant difference in that year between watersheds at $\alpha=0.05$
Table 6 Foliar nutrient concentrations from 1993 to 2006 in red spruce at the BBWM: West Bear Brook (WB) watershed with $\left(\left(\mathrm{NH}_{4}\right)_{2} \mathrm{SO}_{4}\right)$ addition treatment and East
Bear Brook (EB) the reference watershed (data from 1993 to 2003 from Elvir et al. 2005)

\begin{tabular}{|c|c|c|c|c|c|c|c|c|c|c|c|c|c|c|}
\hline \multirow[t]{2}{*}{ Element } & \multicolumn{2}{|l|}{1993} & \multicolumn{2}{|l|}{1998} & \multicolumn{2}{|l|}{1999} & \multicolumn{2}{|l|}{2000} & \multicolumn{2}{|l|}{2002} & \multicolumn{2}{|l|}{2003} & \multicolumn{2}{|l|}{2006} \\
\hline & WB & EB & WB & EB & WB & EB & WB & EB & WB & EB & WB & EB & WB & EB \\
\hline Total N & $1.19^{\mathrm{a}}$ & 1.09 & 1.26 & 1.21 & 1.04 & 1.03 & 1.13 & 1.02 & $1.19^{\mathrm{a}}$ & 1.07 & $1.13^{\mathrm{a}}$ & 1.05 & $1.02^{\mathrm{a}}$ & 0.93 \\
\hline $\mathrm{Ca}$ & 1780 & 1,690 & 1,858 & 2,135 & 1,425 & 1,599 & 1,311 & 1,545 & $1,594^{\mathrm{a}}$ & 2,204 & $1,406^{\mathrm{a}}$ & 1,972 & 1,400 & 1,549 \\
\hline $\mathrm{Mg}$ & $722^{a}$ & 704 & 736 & 853 & 645 & 739 & 656 & 798 & $647^{\mathrm{a}}$ & 768 & $680^{\mathrm{a}}$ & 862 & 709 & 763 \\
\hline K & $7,050^{\mathrm{a}}$ & 7,710 & 7,939 & 8,186 & 7,748 & 7,541 & 8,433 & 8,053 & 7,695 & 7,196 & 6,798 & 6,817 & 5,486 & 5,541 \\
\hline $\mathrm{P}$ & 1,130 & 1,090 & $941^{\mathrm{a}}$ & 1,068 & 883 & 918 & 1,459 & 1,532 & 930 & 909 & 862 & 913 & 796 & 825 \\
\hline $\mathrm{Al}$ & 55.5 & 56.5 & 46.0 & 46.0 & 29.0 & 39.0 & 63.0 & 60.0 & - & 29.0 & 26.0 & 32.0 & 30.0 & 32.0 \\
\hline $\mathrm{Fe}$ & 25.6 & 26.4 & 17.0 & 2.0 & 19.0 & 14.0 & 13.0 & 14.0 & 15.0 & 20.0 & 21.0 & 22.0 & 19.0 & 18.0 \\
\hline $\mathrm{Cu}$ & 3.93 & 3.77 & 6.00 & 5.00 & 1.00 & 0.00 & 3.00 & 4.00 & 3.00 & 3.00 & 3.00 & 3.00 & 3.00 & 3.00 \\
\hline $\mathrm{Mn}$ & 936 & 938 & 704 & 729 & 480 & 445 & 440 & 454 & 379 & 512 & $491^{\mathrm{a}}$ & 879 & 299 & 334 \\
\hline $\mathrm{Zn}$ & 16.5 & 17.5 & 18.0 & 20.0 & 3.0 & 2.0 & 18.0 & 21.0 & 16.0 & 21.0 & 14.0 & 20.0 & $15.0^{\mathrm{a}}$ & 18.0 \\
\hline $\mathrm{Na}$ & & & 63 & 75 & 54 & 68 & 183 & 251 & & & & & & \\
\hline LOI & & & 97 & 97 & 98 & 98 & 97 & 98 & & & & & & \\
\hline $\mathrm{Be}$ & & & & & 0.02 & 0.26 & 0.21 & 0.02 & & & & & & \\
\hline B & 16.5 & 14.9 & & & & & & & 20.0 & 19.0 & 21.0 & 22.0 & $14.0^{\mathrm{a}}$ & 27.0 \\
\hline$\% \mathrm{C}$ & & & & & & & & & & & & & 50 & 49 \\
\hline
\end{tabular}

${ }^{\text {a }}$ Significance between watersheds at $\alpha=0.05$ 
Table 7 Foliar nutrient concentrations from 1993 to 2006 in American beech at the BBWM: West Bear Brook (WB) watershed with $\left(\left(\mathrm{NH}_{4}\right)_{2} \mathrm{SO}_{4}\right)$ addition treatment and East
Bear Brook (EB) the reference watershed (data from 1993 to 2003 from Elvir et al. 2005)

\begin{tabular}{|c|c|c|c|c|c|c|c|c|c|c|c|c|c|c|}
\hline \multirow[t]{2}{*}{ Element } & \multicolumn{2}{|l|}{1993} & \multicolumn{2}{|l|}{1998} & \multicolumn{2}{|l|}{1999} & \multicolumn{2}{|l|}{2000} & \multicolumn{2}{|l|}{2002} & \multicolumn{2}{|l|}{2003} & \multicolumn{2}{|l|}{2006} \\
\hline & $\overline{\mathrm{WB}}$ & EB & WB & $\mathrm{EB}$ & WB & EB & WB & EB & WB & EB & WB & EB & $\mathrm{WB}$ & EB \\
\hline Total N & $2.41^{\mathrm{a}}$ & 2.28 & $2.62^{a}$ & 2.27 & $2.43^{\mathrm{a}}$ & 2.08 & $2.38^{\mathrm{a}}$ & 2.25 & $2.47^{\mathrm{a}}$ & 2.23 & $2.35^{\mathrm{a}}$ & 2.14 & $2.40^{\mathrm{a}}$ & 2.06 \\
\hline $\mathrm{Ca}$ & 4,900 & 5,330 & 6,755 & 7,332 & 4,507 & 5,298 & $5,299^{\mathrm{a}}$ & 7,364 & $3,850^{\mathrm{a}}$ & 5,749 & $3,704^{\mathrm{a}}$ & 5,603 & $3,814^{\mathrm{a}}$ & 5,505 \\
\hline $\mathrm{Mg}$ & 1,610 & 1,550 & $1,443^{\mathrm{a}}$ & 1,720 & 1,547 & 1,474 & $1,561^{\mathrm{a}}$ & 2,060 & $1,006^{\mathrm{a}}$ & 1,573 & $1,038^{\mathrm{a}}$ & 1,648 & $1,186^{\mathrm{a}}$ & 1,600 \\
\hline $\mathrm{K}$ & $7,280^{\mathrm{a}}$ & 8,360 & 6,524 & 7,318 & $6,878^{a}$ & 7,949 & 5,616 & 6,588 & 6,748 & 5,836 & 6,956 & 5,934 & 6,363 & 6,469 \\
\hline $\mathrm{P}$ & 1,270 & 1,180 & $1,288^{\mathrm{a}}$ & 1,025 & $1,511^{\mathrm{a}}$ & 1,061 & 1,277 & 1,178 & $967^{\mathrm{a}}$ & 1,112 & $946^{\mathrm{a}}$ & 1,082 & 1,005 & 1,020 \\
\hline $\mathrm{Al}$ & $19.3^{\mathrm{a}}$ & 16.3 & 32.0 & 24.0 & 37.0 & 33.0 & 45.0 & 60.0 & - & - & 19.0 & 21.0 & 14.0 & 22.0 \\
\hline $\mathrm{Fe}$ & 61.8 & 60.1 & 5.0 & 11.0 & 52.0 & 35.0 & 14.0 & 41.0 & 46.0 & 40.0 & 43.0 & 46.0 & 49.0 & 46.0 \\
\hline $\mathrm{Cu}$ & $6.54^{\mathrm{a}}$ & 5.80 & 8.00 & 8.00 & 7.00 & 6.00 & 6.00 & 6.00 & 5.00 & 6.00 & 7.00 & 7.00 & 5.00 & 5.00 \\
\hline $\mathrm{Mn}$ & $849^{a}$ & 656 & $1,319^{\mathrm{a}}$ & 881 & $939^{a}$ & 667 & $1,187^{\mathrm{a}}$ & 951 & 579 & 576 & 727 & 860 & 430 & 437 \\
\hline $\mathrm{Zn}$ & 23.8 & 21.4 & 19.0 & 25.0 & 25.0 & 27.0 & 27.0 & 26.0 & 16.0 & 19.0 & 17.0 & 21.0 & 18.0 & 23.0 \\
\hline $\mathrm{Na}$ & & & 114 & 49 & 78 & 28 & 139 & 266 & & & & & & \\
\hline LOI & & & 95 & 94 & 96 & 95 & 96 & 95 & & & & & & \\
\hline $\mathrm{Be}$ & & & & & 0.00 & 0.00 & 0.25 & 0.24 & & & & & & \\
\hline B & 24.0 & 24.6 & & & & & & & 30.0 & 32.0 & 29.0 & 35.0 & 24.0 & 28.0 \\
\hline$\% \mathrm{C}$ & & & & & & & & & & & & & 49 & 49 \\
\hline
\end{tabular}

${ }^{\text {a }}$ Significance between watersheds at $\alpha=0.05$

for WB. Elvir et al. (2003) and this study indicate significant annual differences in BAI between watersheds since the treatment began (1989) until 1997, with higher BAI in trees in the treated WB. The higher growth in WB trees declined after 1997, and from 1999 to 2005, BAI growth was similar in both watersheds (Fig. 4).

Table 8 Foliar nutrient concentrations from 1993 to 2006 in sugar maple at the BBWM: West Bear Brook (WB) watershed with $\left(\left(\mathrm{NH}_{4}\right)_{2} \mathrm{SO}_{4}\right)$ addition treatment and East
Foliar chemistry

Foliar $\mathrm{N}$ concentrations were significantly higher in the treated WB than in the reference EB for all tree species, except in 1998, 1999, and 2000 when concentrations between watersheds were similar for red spruce (Tables 6, 7, and 8). Among species,

\begin{tabular}{|c|c|c|c|c|c|c|c|c|c|c|c|c|c|c|}
\hline \multirow[t]{2}{*}{ Element } & \multicolumn{2}{|l|}{1993} & \multicolumn{2}{|l|}{1998} & \multicolumn{2}{|l|}{1999} & \multicolumn{2}{|l|}{2000} & \multicolumn{2}{|l|}{2002} & \multicolumn{2}{|l|}{2003} & \multicolumn{2}{|l|}{2006} \\
\hline & WB & EB & WB & EB & WB & EB & WB & EB & WB & EB & WB & EB & WB & EB \\
\hline Total N & $2.08^{a}$ & 1.56 & $2.19^{\mathrm{a}}$ & 1.71 & $1.97^{\mathrm{a}}$ & 1.61 & $2.01^{\mathrm{a}}$ & 1.85 & $1.94^{\mathrm{a}}$ & 1.73 & $1.95^{\mathrm{a}}$ & 1.77 & $1.91^{\mathrm{a}}$ & 1.74 \\
\hline $\mathrm{Ca}$ & $6,090^{\mathrm{a}}$ & 7,450 & 6,995 & 7,369 & 5,759 & 5,997 & 7,327 & 6,315 & 3,662 & 3,751 & 3,041 & 3,072 & 2,732 & 2,879 \\
\hline $\mathrm{Mg}$ & 1,020 & 1,110 & 1,001 & 1,016 & 1,045 & 901 & 1,256 & 1,030 & 571 & 714 & 635 & 720 & 533 & 611 \\
\hline K & 8,310 & 8,130 & 8,837 & 9,264 & 7,974 & 8,103 & 7,856 & 7,632 & 7,674 & 7,030 & 7,408 & 7,143 & 6,557 & 6,132 \\
\hline $\mathrm{P}$ & $1,060^{\mathrm{a}}$ & 907 & $1,095^{\mathrm{a}}$ & 937 & 1,078 & 971 & $1,884^{\mathrm{a}}$ & 1,268 & 983 & 952 & 978 & 1,010 & 1,040 & 1,087 \\
\hline $\mathrm{Al}$ & $24.6^{\mathrm{a}}$ & 14.9 & 41.0 & 15.0 & 46.0 & 72.0 & 58.0 & 110.0 & - & - & 20.0 & 21.0 & 20.0 & 20.0 \\
\hline $\mathrm{Fe}$ & $59.0^{\mathrm{a}}$ & 43.5 & 25.0 & 1.0 & 63.0 & 55.0 & 52.0 & 27.0 & 38.0 & 34.0 & 44.0 & 39.0 & 43.0 & 42.0 \\
\hline $\mathrm{Cu}$ & 5.38 & 5.01 & 6.00 & 8.00 & 5.00 & 5.00 & 7.00 & 6.00 & 6.00 & 7.00 & 6.00 & 7.00 & 5.00 & 6.00 \\
\hline $\mathrm{Mn}$ & 940 & 769 & $1,329^{\mathrm{a}}$ & 868 & $1,316^{\mathrm{a}}$ & 701 & $1,491^{\mathrm{a}}$ & 787 & 738 & 664 & 1,046 & 1,196 & 571 & 624 \\
\hline $\mathrm{Zn}$ & 21.5 & 20.0 & 27.0 & 20.0 & 17.0 & 35.0 & 35.0 & 29.0 & 15.0 & 17.0 & 15.0 & 16.0 & 14.0 & 15.0 \\
\hline $\mathrm{Na}$ & & & 133 & 133 & 113 & 88 & 423 & 202 & & & & & & \\
\hline LOI & & & 95 & 94 & 95 & 95 & 95 & 95 & & & & & & \\
\hline $\mathrm{Be}$ & & & & & 0.00 & 0.00 & 0.13 & 0.03 & & & & & & \\
\hline B & 40.9 & 37.6 & & & & & & & 40.0 & 41.0 & 48.0 & 48.0 & 38.0 & 36.0 \\
\hline$\% \mathrm{C}$ & & & & & & & & & & & & & 50 & 49 \\
\hline
\end{tabular}

${ }^{\text {a }}$ Significance between watersheds at $\alpha=0.05$

Bear Brook (EB) the reference watershed (data for 1993 to 2003 from Elvir et al. 2005) 
Table 9 Sugar maple, American beech, and red spruce net photosynthetic and transpiration rates at the BBWM (standard deviations in parentheses; from Elvir et al. 2006; reprinted with permission from Forest Ecology and Management, vol. 221, copyright 2006 by Elsevier)

\begin{tabular}{|c|c|c|c|c|}
\hline & \multicolumn{2}{|c|}{ East Bear Watershed (reference) } & \multicolumn{2}{|c|}{ West Bear Watershed (treated) } \\
\hline & 2002 & 2003 & 2002 & 2003 \\
\hline \multicolumn{5}{|l|}{ Sugar maple } \\
\hline Photosynthesis $\left(\mu \mathrm{m} / \mathrm{m}^{2} / \mathrm{s}\right)$ & $7.31(1.03)$ & $7.57(1.18)$ & $8.80(1.61)^{\mathrm{a}}$ & $8.57(1.13)^{\mathrm{a}}$ \\
\hline Transpiration $\left(\mathrm{mm} / \mathrm{m}^{2} / \mathrm{s}\right)$ & $1.74(0.30)$ & $1.83(0.27)$ & $2.01(0.50)$ & $1.80(0.38)$ \\
\hline \multicolumn{5}{|l|}{ American beech } \\
\hline Photosynthesis $\left(\mu \mathrm{m} / \mathrm{m}^{2} / \mathrm{s}\right)$ & $7.15(1.39)$ & $8.70(2.20)$ & $7.73(1.29)$ & $8.28(1.52)$ \\
\hline Transpiration $\left(\mathrm{mm} / \mathrm{m}^{2} / \mathrm{s}\right)$ & $1.84(0.30)^{\mathrm{b}}$ & $2.35(0.33)$ & $1.72(0.38)^{b}$ & $2.08(0.30)$ \\
\hline \multicolumn{5}{|l|}{ Red spruce } \\
\hline Photosynthesis $\left(\mu \mathrm{m} / \mathrm{m}^{2} / \mathrm{s}\right)$ & $7.40(1.87)^{\mathrm{b}}$ & $9.57(1.15)$ & $8.19(1.97)$ & $9.48(1.55)$ \\
\hline Transpiration $\left(\mathrm{mm} / \mathrm{m}^{2} / \mathrm{s}\right)$ & $1.24(0.37)$ & $1.57(0.44)$ & $1.56(0.79)$ & $1.73(0.75)$ \\
\hline
\end{tabular}

aSignificance between watersheds at the 0.05 level

${ }^{\mathrm{b}}$ Significance between years within a watershed at the 0.05 level

American beech had the highest foliar $\mathrm{N}$ concentrations in both watersheds. Foliar $\mathrm{Ca}$ and $\mathrm{Mg}$ concentrations were contrasting among species. Except in 1993 when foliar Ca was significantly lower in trees growing in $\mathrm{WB}$, foliar $\mathrm{Ca}$ and $\mathrm{Mg}$ were similar between watersheds for sugar maple. Foliar $\mathrm{Ca}$ and $\mathrm{Mg}$ concentrations in American beech and red spruce were significantly lower in
Table 10 Frequency and abundance of plant species $<0.6 \mathrm{~m}$ in height in the deciduous forest types at BBWM in 2005 (from Kenlan 2006). West Bear Brook (WB) watershed with $\left(\left(\mathrm{NH}_{4}\right)_{2} \mathrm{SO}_{4}\right)$ addition treatment and East Bear Brook (EB) the reference watershed

*Significance between watersheds at the 0.05 level

\begin{tabular}{|c|c|c|c|c|c|c|}
\hline \multirow[t]{2}{*}{ Species } & \multicolumn{3}{|c|}{$\begin{array}{l}\text { Abundance } \\
\text { (average percent cover) }\end{array}$} & \multicolumn{3}{|c|}{$\begin{array}{l}\text { Frequency } \\
\text { (percent of plots) }\end{array}$} \\
\hline & EB & WB & $p$ value & $\mathrm{EB}$ & WB & $p$ value \\
\hline Abies balsamea & 0.16 & 0.00 & 0.187 & 7 & 0 & 0.184 \\
\hline Aralia nudicaulis & 0.60 & 0.97 & 0.276 & 13 & 15 & 0.530 \\
\hline Acer pensylvanicum & 1.11 & 0.24 & $0.001 *$ & 58 & 24 & $0.002 *$ \\
\hline Acer rubrum & 0.71 & 0.48 & 0.203 & 53 & 36 & 0.101 \\
\hline Acer saccharum & 0.49 & 0.55 & 0.443 & 31 & 18 & 0.147 \\
\hline Aster spp. & 1.53 & 0.45 & $0.029 *$ & 40 & 15 & $0.015^{*}$ \\
\hline Betula alleghaniensis & 0.49 & 0.27 & 0.248 & 22 & 15 & 0.144 \\
\hline Cornus canadensis & 0.02 & 0.00 & 0.572 & 2 & 0 & 0.582 \\
\hline Cypripedium spp. & 0.13 & 0.00 & 0.317 & 4 & 0 & 0.325 \\
\hline Fern & 7.29 & 1.15 & 0.066 & 24 & 18 & 0.351 \\
\hline Fagus grandifolia & 0.40 & 0.27 & 0.341 & 22 & 15 & 0.320 \\
\hline Fragaria virginiana & 0.04 & 0.00 & 0.324 & 4 & 0 & 0.323 \\
\hline Graminoids & 0.22 & 0.39 & 0.358 & 13 & 12 & 0.591 \\
\hline Hamamelis virginiana & 0.36 & 0.64 & 0.316 & 7 & 9 & 0.499 \\
\hline Lycopodium spp. & 0.33 & 0.00 & 0.331 & 4 & 0 & 0.324 \\
\hline Maianthemum canadensis & 0.36 & 0.24 & 0.339 & 27 & 12 & 0.097 \\
\hline Medeola virginiana & 0.11 & 0.00 & 0.587 & 2 & 0 & 0.570 \\
\hline Polygonatum pubescens & 0.02 & 0.06 & 0.384 & 2 & 6 & 0.392 \\
\hline Picea rubens & 0.58 & 0.67 & 0.424 & 31 & 27 & 0.449 \\
\hline Pinus strobus & 0.02 & 0.00 & 0.580 & 2 & 0 & 0.584 \\
\hline Rubus spp. & 0.07 & 0.61 & 0.427 & 7 & 3 & 0.426 \\
\hline Sorbus americana & 0.02 & 0.06 & 0.389 & 2 & 6 & 0.384 \\
\hline Trientalis borealis & 0.27 & 0.06 & 0.098 & 18 & 6 & 0.115 \\
\hline Uvularia sessilifolia & 1.76 & 0.91 & 0.123 & 31 & 27 & 0.456 \\
\hline Viola spp. & 0.13 & 0.00 & 0.326 & 4 & 0 & 0.332 \\
\hline Vaccinium spp. & 0.00 & 0.15 & 0.423 & 0 & 3 & 0.421 \\
\hline
\end{tabular}


Table 11 Mean biomass per plot for all species combined at BBWM in 2005 (from Kenlan 2006). West Bear Brook (WB) watershed with $\left(\left(\mathrm{NH}_{4}\right)_{2} \mathrm{SO}_{4}\right)$ addition treatment and East Bear Brook (EB) the reference watershed

\begin{tabular}{|c|c|c|c|c|c|}
\hline \multirow[t]{2}{*}{ Cover type } & \multicolumn{2}{|c|}{$\begin{array}{l}\text { Biomass } \\
\left(\text { dry } \mathrm{g} / \mathrm{m}^{2}\right)\end{array}$} & \multicolumn{2}{|c|}{$\begin{array}{l}\text { Biomass } \\
\left(\text { dry } \mathrm{g} / \mathrm{m}^{2}\right)\end{array}$} & \multirow[t]{2}{*}{$p$ value $^{\mathrm{b}}$} \\
\hline & $\mathrm{EB}^{\mathrm{a}}$ & $\mathrm{WB}^{\mathrm{a}}$ & $\mathrm{EB}^{\mathrm{b}}$ & $\mathrm{WB}^{\mathrm{b}}$ & \\
\hline Mixed wood & 2.62 & 2.02 & 0.35 & 0.10 & 0.141 \\
\hline Hardwood & 3.13 & 2.09 & 0.92 & 0.24 & 0.262 \\
\hline Combined & 2.74 & 2.05 & 0.43 & 0.15 & 0.107 \\
\hline
\end{tabular}

No significances were found between watersheds at the 0.05 level

a Arithmetic means calculated from untransformed data

${ }^{\mathrm{b}}$ Geometric means and significance tests following $\log$ transformation to correct non-normal data

trees growing in the treated WB, except in 1998, 1999, and 2000 in red spruce where differences were not significant. Species had similar foliar $\mathrm{K}$ concentrations between watersheds, except in 1993 for red spruce and 1999 for American beech when foliar $\mathrm{K}$ concentrations were significantly lower in the treated WB watershed.

Foliar trends showed a Ca decline especially in WB in all species. From 1993 to 2006, foliar Ca declined by $-21 \%$ in $\mathrm{WB}$ and $-8 \%$ in $\mathrm{EB}$ for red spruce, $-22 \%$ in $\mathrm{WB}$ and $-3 \%$ in $\mathrm{EB}$ for American beech, and $-55 \%$ in $\mathrm{WB}$ and $-61 \%$ in EB for sugar maple. Similarly to $\mathrm{Ca}$, foliar $\mathrm{K}$ concentrations followed a similar declining trend in both watersheds in all species. From 1993 to 2006 , foliar K declined by $-22 \%$ in WB and $-28 \%$ in $\mathrm{EB}$ for red spruce, $-13 \%$ in $\mathrm{WB}$ and $-23 \%$ in $\mathrm{EB}$ for American beech, and $-21 \%$ in WB and $-25 \%$ in $\mathrm{EB}$ for sugar maple. However, $\mathrm{Mg}$ concentrations declined from 1993 to 2006 only in American beech $(-26 \%$ in WB) and sugar maple $(-48 \%$ in $\mathrm{WB}$ and $-45 \%$ in $\mathrm{EB})$.

Physiology

Many studies provide consistent reports of the effects of foliar $\mathrm{Ca}$ and $\mathrm{Mg}$ on physiological processes (Dreyer et al. 1994; Laing et al. 2000; Mclaughlin et al. 1990; Mehne-Jakobs 1995, 1996; Oren et al. 1993; Weikert et al. 1989). At the BBWM, Elvir et al. (2006) reported that only sugar maple in WB had higher photosynthetic rates than trees in the reference watershed and showed a significant positive correlation between photosynthetic rates and foliar $\mathrm{N}$ concentration (Table 9). Photosynthetic rates were similar between watersheds for red spruce and American beech. Elvir et al. (2006) suggested that the lack of response in photosynthesis to higher foliar $\mathrm{N}$ in American beech and red spruce might be due to $\mathrm{Ca}$ and/or $\mathrm{Mg}$ limitations, as indicated by their lower concentrations in the foliage of treated trees. Reported photosynthetic rates range from 5 to $9 \mu \mathrm{mol} / \mathrm{m}^{2} / \mathrm{s}$ for mature sugar maple (Amthor et al. 1990; Clair and Lynch 2005), 6 to $9 \mu \mathrm{mol} / \mathrm{m}^{2} / \mathrm{s}$ for mature American beech (Amthor et al. 1990), $7.64 \mu \mathrm{mol} / \mathrm{m}^{2} / \mathrm{s}$ for $\geq 53$ years old red spruce
Table 12 Foliar nutrient concentrations of Acer saccharum seedlings and Uvularia sessifolia at the BBWM in 2005 (total $\mathrm{N}$ in \%, other nutrients in ppm) (from Kenlan 2006).
West Bear Brook (WB) watershed with $\left(\left(\mathrm{NH}_{4}\right)_{2} \mathrm{SO}_{4}\right)$ addition treatment and East Bear Brook (EB) the reference watershed

\begin{tabular}{|c|c|c|c|c|c|c|}
\hline \multirow[t]{2}{*}{ Nutrient } & \multicolumn{2}{|c|}{ Acer saccharum } & \multirow[t]{2}{*}{$p$ value } & \multicolumn{2}{|c|}{ Uvularia sessilifolia } & \multirow[t]{2}{*}{$p$ value } \\
\hline & $\mathrm{EB}$ & WB & & $\mathrm{EB}$ & WB & \\
\hline $\mathrm{TN}$ & 2.0 & 2.4 & $0.000 *$ & 2.13 & 2.35 & 0.057 \\
\hline $\mathrm{Ca}$ & 9,570 & 6,740 & $0.002 *$ & 10,260 & 9,560 & 0.132 \\
\hline $\mathrm{Mg}$ & 1,430 & 1,280 & 0.095 & 2,170 & 2,060 & 0.558 \\
\hline K & 6,770 & 11,010 & $0.000 *$ & 15,200 & 12,350 & $0.003^{*}$ \\
\hline $\mathrm{P}$ & 1,040 & 1,200 & $0.000 *$ & 1,260 & 1,240 & 0.670 \\
\hline $\mathrm{Al}$ & 39.4 & 44.0 & 0.583 & 69.7 & 61.7 & 0.247 \\
\hline B & 43.6 & 48.9 & 0.157 & 22.6 & 21.9 & 0.385 \\
\hline $\mathrm{Cu}$ & 5.69 & 6.15 & 0.522 & 8.74 & 10.53 & $0.006^{*}$ \\
\hline $\mathrm{Fe}$ & 53.6 & 49.0 & 0.390 & 71.7 & 69.6 & 0.814 \\
\hline $\mathrm{Mn}$ & 1,272 & 1,302 & 0.943 & 449 & 445 & 0.934 \\
\hline $\mathrm{Zn}$ & 35.5 & 36.3 & 0.812 & 17.4 & 21.3 & $0.013 *$ \\
\hline
\end{tabular}

*Significance between watersheds at the 0.05 level 
Table 13 Mensurational data from the 2-4 m size class sugar maple saplings (from Bethers et al. 2009). Reprinted with permission from Forest Ecology and Management, vol. 258, copyright 2009 by Elsevier

\begin{tabular}{llll}
\hline & East Bear-reference & \multicolumn{1}{c}{$\frac{t \text { West Bear-treated }}{p \text { values }}$} \\
\cline { 2 - 3 } & Mean $(\mathrm{SE})$ & $8.0(3.3)$ & 0.09 \\
Saplings per plot & $1.8(0.54)$ & $358(146)$ & 0.08 \\
Saplings per ha & $81(24)$ & $2.60(0.03)$ & 0.55 \\
Sapling height $(\mathrm{m})$ & $2.60(0.02)$ & $10.5(0.2)$ & 0.95 \\
Sapling DBH $(\mathrm{mm})$ & $10.6(0.2)$ & $17.0(0.3)$ & 0.22 \\
Sapling basal diameter $(\mathrm{mm})$ & $17.8(0.2)$ & $18.6(1.3)$ & $0.55^{\mathrm{a}}$ \\
Plot \% canopy openness & $17.9(1.5)$ & $12.0(0.4)$ & 0.76 \\
Sapling \% canopy openness & $12.3(0.5)$ & & \\
\hline
\end{tabular}

Means based on 84 plots and 422 saplings

$S E$ standard error at the BBWM

No significances were found between watersheds at the 0.05 level

${ }^{a}$ Based on log transformation

(Ward 2001), values consistent with results reported for these species at the BBWM.

\section{Ground flora}

Kenlan (2006) found 26 plant species within forest floor plots on EB and WB combined. Of these, 25 species occurred in EB and 20 in WB. Seven species (Fragaria virginiana, Cypripedium spp., Viola spp., Medeola virginiana, Cornus canadensis, Pinus strobus, and Lycopodium spp.) occurred only in EB, and two species (Polygonum convolvulus and Vaccinium spp.) occurred only in WB (Table 10). The total aboveground biomass was similar between watersheds for mixed woods and hardwoods and their combination (Table 11).

Kenlan (2006) showed that foliar nutrient concentrations were significantly different between watersheds for only one of the two species studied: A. saccharum and $U$. sessilifolia. For $A$. saccharum seedlings, foliar $\mathrm{N}$ was higher and foliar $\mathrm{Ca}$ was lower in WB than EB (Table 12).

\section{Sugar maple saplings}

Bethers et al. (2009) showed that sugar maple sapling density was not significantly different between WB and EB, most likely due to high withinwatershed variability for this species (Table 13). Sapling height, DBH, and basal diameter were similar across watersheds. Positive correlations between height and DBH were strong in both watersheds $\left(R^{2}=0.63, p<0.001\right)$. Bethers et al. (2009) also reported similar canopy openness of $17.9 \%$ and $18.6 \%$ in $\mathrm{EB}$ and $\mathrm{WB}$, respectively (Table 13). Photographs, taken above the leader of each sapling, also showed similar light environments for saplings across treatments with $12.3 \%$ and $12.0 \%$ openness in $\mathrm{EB}$ and $\mathrm{WB}$, respectively. No significant correlation was found

Table 14 Foliar nutrient concentrations for sugar maple saplings in 2008 (from Bethers et al. 2009). Reprinted with permission from Forest Ecology and Management, vol. 258, copyright 2009 by Elsevier

\begin{tabular}{|c|c|c|c|}
\hline & $\begin{array}{l}\text { East Bear- } \\
\text { untreated }\end{array}$ & $\begin{array}{l}\text { West Bear- } \\
\text { treated }\end{array}$ & $t$ test \\
\hline & Mean (SE) & Mean (SE) & $p$ values \\
\hline $\mathrm{C}$ & $47.6(0.1)$ & $47.8(0.1)$ & 0.272 \\
\hline $\mathrm{N}$ & $2.03(0.04)$ & $2.38(0.03)$ & $<0.001 *$ \\
\hline $\mathrm{P}$ & $1,102(20)$ & $1,230(27)$ & $<0.001^{*}, \mathrm{a}$ \\
\hline K & $8,274(291)$ & $9,736(316)$ & $0.001 *$ \\
\hline $\mathrm{Ca}$ & $6,922(312)$ & $5,367(262)$ & $<0.001^{*}, \mathrm{a}$ \\
\hline $\mathrm{Mg}$ & $1,380(54)$ & $1,253(41)$ & 0.066 \\
\hline $\mathrm{Mn}$ & $965(60)$ & $1,064(76)$ & $0.323^{\mathrm{a}}$ \\
\hline $\mathrm{Al}$ & $16.9(1.5)$ & $29.9(1.5)$ & $<0.001^{*}, \mathrm{a}$ \\
\hline $\mathrm{Fe}$ & $43.1(1.2)$ & $47.2(1.0)$ & $0.008^{*}, \mathrm{a}$ \\
\hline B & $42.9(1.4)$ & $47.3(1.9)$ & 0.066 \\
\hline $\mathrm{Cu}$ & $6.0(0.2)$ & $6.1(0.2)$ & 0.726 \\
\hline $\mathrm{Zn}$ & $23.8(1.6)$ & $23.5(0.7)$ & $0.574^{\mathrm{a}}$ \\
\hline
\end{tabular}

Untreated $n=36 ;\left(\left(\mathrm{NH}_{4}\right)_{2} \mathrm{SO}_{4}\right)$-treated $n=37$. $\mathrm{C}$ and $\mathrm{N}$ are percent by mass, other nutrients are in parts per million

$S E$ standard error at the BBWM

*Significance between watersheds at the 0.05 level

${ }^{\text {a }}$ Based on log transformation of the data 


\section{A/Ci Curve Analysis}

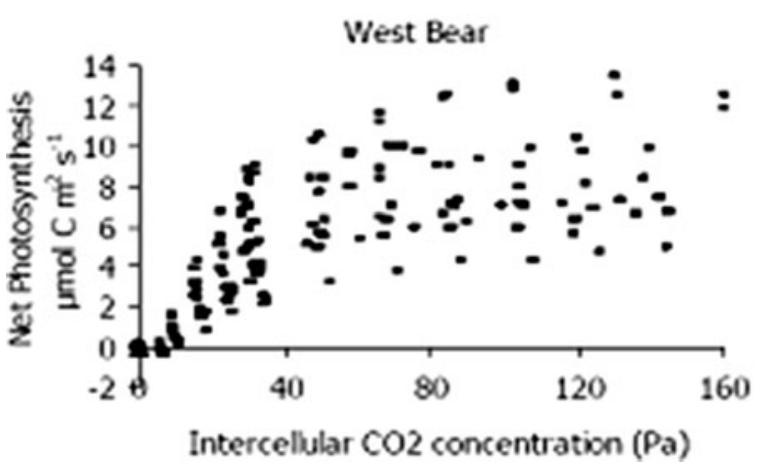

Fig. 5 Individual $A-C_{i}$ curves at the BBWM. Untreated $n=12 ;\left(\left(\mathrm{NH}_{4}\right)_{2} \mathrm{SO}_{4}\right)$-treated $n=14$. West Bear Brook $(W B)$ watershed with $\left(\left(\mathrm{NH}_{4}\right)_{2} \mathrm{SO}_{4}\right)$ addition treatment and East Bear Brook $(E B)$ the reference watershed (from

between sugar maple sapling density and canopy openness $\left(R^{2}=0.0017, p=0.72\right)$.

Foliar chemical data of sugar maple saplings in Bethers et al. (2009) add consistent evidence that the $\mathrm{N}+\mathrm{S}$ addition treatment has increased $\mathrm{N}$, decreased base cation concentrations, and altered $\mathrm{P}, \mathrm{Fe}$, and $\mathrm{Al}$ availability in WB. Consis-

Table 15 Photosynthetic parameters for sugar maple saplings at BBWM. West Bear Brook (WB) watershed with $\left(\left(\mathrm{NH}_{4}\right)_{2} \mathrm{SO}_{4}\right)$ addition treatment and East Bear Brook (EB) the reference watershed (from Bethers et al. 2009). Reprinted with permission from Forest Ecology and Management, vol. 258, copyright 2009 by Elsevier

\begin{tabular}{|c|c|c|c|}
\hline & $\begin{array}{l}\text { East Bear- } \\
\text { untreated }\end{array}$ & $\begin{array}{l}\text { West Bear- } \\
\text { treated }\end{array}$ & $t$ test \\
\hline & Mean (SE) & $\overline{\text { Mean (SE) }}$ & $\overline{p \text { values }}$ \\
\hline $\mathrm{Vc}_{\max }$ & $19.40(1.03)$ & $14.73(1.26)$ & $0.010^{\mathrm{a}}$ \\
\hline$J_{\max }$ & $50.38(1.92)$ & $42.98(2.58)$ & $0.035^{\mathrm{a}}$ \\
\hline TPU & $3.54(0.10)$ & $3.08(0.18)$ & $0.044^{\mathrm{a}}$ \\
\hline$A_{\text {net }}$ & $5.82(0.29)$ & $5.79(0.50)$ & 0.956 \\
\hline Resp & $1.12(0.05)$ & $0.94(0.06)$ & $0.044^{\mathrm{a}}$ \\
\hline Cond & $0.10(0.03)$ & $0.10(0.03)$ & 0.534 \\
\hline LMA & $31.8(0.86)$ & $32.1(0.62)$ & 0.696 \\
\hline
\end{tabular}

Untreated $n=12 ;\left(\left(\mathrm{NH}_{4}\right)_{2} \mathrm{SO}_{4}\right)$-treated $n=14$. Units are in micromoles per square meter per second, except LMA in grams per square meter and Cond in moles per square meter

$V c_{\max }$ carboxylation capacity, $J_{\max }$ electron transport capacity, TPU triose phosphate utilization, $A_{\text {net }}$ lightsaturated net photosynthesis, Resp respiration, Cond stomatal conductivity to $\mathrm{CO}_{2}, L M A$ leaf mass per area

${ }^{a}$ Significance between watersheds at the 0.05 level
East Bear

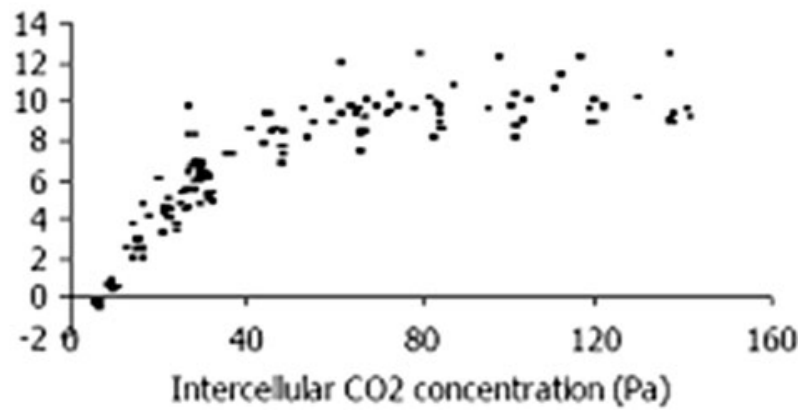

Bethers et al. 2009). Reprinted with permission from Forest Ecology and Management, vol. 258, copyright 2009 by Elsevier

tent with previously presented tree and seedling foliar chemistry, foliar $\mathrm{N}$ concentrations of sugar maple saplings were significantly higher in WB than in EB. In contrast, foliar Ca concentrations were higher in EB than in WB. Similar to the results found for sugar maple seedlings, sugar maple sapling foliar $\mathrm{K}$ and $\mathrm{P}$ concentrations were significantly higher in WB than foliar sapling concentrations in EB (Table 14). Foliar Al and Fe concentrations were also significantly higher in WB than EB.

Bethers et al. (2009) showed that sugar maple saplings growing in the treated WB watershed had highly variable net photosynthesis $(A)$ as intercellular $\mathrm{CO}_{2}\left(C_{i}\right)$ increased, while sugar maple saplings growing in the reference EB watershed had much less variability along the $A-C_{i}$ curve (Fig. 5). Also, photosynthetic capacity, $V_{c_{\max }}$, $J_{\max }$, TPU, respiration, and leaf temperature were ssssslower in saplings growing in WB than those in EB (Table 15). Higher sapling leaf temperature in $\mathrm{EB}$ was positively correlated with $\mathrm{Vc}_{\max }\left(R^{2}=\right.$ $0.503, p=0.0001)$ and more weakly correlated with $J_{\max }\left(R^{2}=0.189, p=0.015\right)$ and TPU $\left(R^{2}=\right.$ 0.155, $p=0.026$; Bethers et al. 2009).

\section{Discussion}

The results of this study on the effects of simulated acid deposition on the forest at the WB 
show that, overall, the treatment has not affected stand dynamics and stand growth at the watershed level. Tree density, mortality, and DBH growth from standard survey measurements at the BBWM are not different between the treated WB watershed and the reference EB watershed. The forest structure and tree species richness are similar between watersheds. Tree data for 20002008 showed a decline in density in both watersheds, which is characteristic of the successional stage of this forest. As forests get older and trees grow larger, density tends to drop due to the mortality of suppressed trees. Repeated DBH measurements of the same trees over a 9-year period indicated that forest growth was not significantly affected by the treatment compared to reference trees.

Kenlan (2006) showed that forest floor species diversity and aboveground biomass were similar between watersheds. Bethers et al. (2009) also showed no differences between watersheds in density, biomass, canopy openness, DBH, and height in sugar maple seedlings. Saplings studied by Bethers et al. (2009) were mostly 17-19 years old, indicating that their life span was within the treatment period (1989-2006). The results suggest that the treatment at the stand level has not affected the ability of sugar maple to regenerate and grow since treatments started.

There is no evidence that the treatment has affected forest growth or biomass accumulation at the watershed level. Studies have suggested that $\mathrm{N}$ deposition might induce changes in tree species composition, but those changes may only appear after longer periods of chronic exposure than the 12 years of this study. At the BBWM, effects of the treatment on species density or species richness might appear after a longer time of treatment.

Evidence shows that the treatment has undoubtedly induced foliar chemistry changes at the BBWM. Foliar N concentrations have remained significantly higher in seedlings, saplings, and trees on the treated watershed for all species. Foliar N concentrations increased in WB since the early years of the treatment. Weber and Wiersma (1997) showed that $\mathrm{N}$ concentrations were significantly higher in two moss species (Dicranum fulvum and Bazzania trilobata) after 3 years of treatment on WB watershed compared with the reference EB watershed. Tree foliar N concentrations were significantly higher in the WB after 4 years of treatment (White et al. 1999). Consecutive sampling of tree foliage reported by Elvir et al. (2005) and this study and sampling of forest floor vegetation (Kenlan 2006) and sapling foliage (Bethers et al. 2009) have consistently confirmed that the $\mathrm{N}+\mathrm{S}$ addition treatment has induced significant foliar $\mathrm{N}$ increases.

On the other hand, these same foliar studies conducted at the BBWM suggest that overall base cation availability for plant uptake has declined in the treated watershed, consistent with soil base cation depletion at the treated WB reported by Fernandez et al. (1999, 2003), Jefts et al. (2004), and recently by SanClements (2008). Foliar chemistry shows that $\mathrm{Ca}$ and $\mathrm{Mg}$ have declined in mosses (Weber and Wiersma 1997), red spruce and American beech trees (Elvir et al. 2005 and this study), sugar maple seedlings and $U$. sessilifolia (Kenlan 2006), and sugar maple saplings (Bethers et al. 2009) in the treated watershed.

In contrast, foliar $\mathrm{Ca}$ and $\mathrm{Mg}$ concentrations were similar between watersheds for sugar maple trees. Kolb and McCormick (1993) indicated that foliar $\mathrm{Ca}$ and $\mathrm{Mg}$ concentrations for healthy sugar maple trees range between 5,000 and 21,900 ppm for $\mathrm{Ca}, 1,100$ and 4,000 ppm for $\mathrm{Mg}$, and 5,500 and 10,400 ppm for K. Foliar $\mathrm{Ca}$ and $\mathrm{Mg}$ concentrations have fallen below these ranges for seemingly healthy sugar maples at the BBWM. Foliar Ca and $\mathrm{Mg}$ concentrations had a severe drop from 2000 to 2002 in sugar maple in both watersheds and for foliar $\mathrm{Ca}$ and $\mathrm{Mg}$ in American beech in the WB watershed. Although time of sample collection varied among studied years $(2002,2003$, and 2006; foliar samples were collected late July or early August, while in earlier years, samples were collected in early September), it appears to have no major effects on foliar concentrations. In a separate study on sugar maple condition at the BBWM, sugar maple foliage was sampled again in early September 2008. While these data are still being analyzed, preliminary results tend to confirm the steady decline in $\mathrm{Ca}$ and $\mathrm{Mg}$ in sugar maple foliage on both watersheds. Mclaughlin and Wimmer (1999) indicated that Ca mobility is low, making $\mathrm{Ca}$ uptake and distribution rate a limiting process. Schaberg et al. (2006) showed that 
Ca concentrations of sugar maple foliar samples collected from 14 sites over a 3-week period in Vermont ranged from 3,600 to $15,300 \mathrm{ppm}$. They recognized that time of sample collection might affect concentrations and they suggested that differences among sites were more strongly explained by soil $\mathrm{Ca}$ availability where sites richer in soil $\mathrm{Ca}$ had higher foliar $\mathrm{Ca}$ concentrations. Also, Horsley et al. (2000) reported that foliar Mg concentrations below $\sim 700$ ppm were associated with declining sugar maple stands.

The foliar $\mathrm{Ca}$ and $\mathrm{Mg}$ data at the Bear Brook site suggest that sugar maple may have experienced shortages in $\mathrm{Ca}$ and $\mathrm{Mg}$ supply in both $\mathrm{EB}$ and $\mathrm{WB}$, yet no differences between the treated and reference watersheds were found. Results, however, showed that the treatment has increased $\mathrm{N}$ availability, reflected in higher foliar $\mathrm{N}$ concentrations in sugar maple trees growing in the treated watershed. The decline in $\mathrm{Ca}$ and $\mathrm{Mg}$ concentrations in both watersheds indicate that sugar maple, although a deep-rooted species (Godman et al. 1990) and growing in richer soils (Fernandez et al. 1999), is experiencing a greater depletion of exchangeable $\mathrm{Ca}$ and $\mathrm{Mg}$ in both watersheds than the other species.

Although foliar $\mathrm{K}$ concentrations were similar between watersheds for sugar maple, beech, and red spruce trees $>10 \mathrm{~cm} \mathrm{DBH}$, foliar $\mathrm{K}$ concentrations in seedlings (Kenlan 2006) and saplings (Bethers et al. 2009) were higher in the treated than the reference watershed. In a recent soil chemistry study at the BBWM, SanClements (2008) showed that, although soil $\mathrm{Ca}$ and $\mathrm{Mg}$ concentrations were lower (especially at the upper soil horizons for hardwoods) at the WB compared with $\mathrm{EB}$, soil $\mathrm{K}$ concentrations in the hardwood stands were similar between watersheds. The lower soil $\mathrm{Ca}$ and $\mathrm{Mg}$ and no detectable changes in soil $\mathrm{K}$ in $\mathrm{WB}$ suggest that roots at the upper soil level might uptake more $\mathrm{K}$ passively as $\mathrm{Ca}$ and $\mathrm{Mg}$ become less available. While no clear effect of the treatment was detected for the other foliar nutrients in trees, results for seedlings and saplings suggest that foliar $\mathrm{P}, \mathrm{Al}$, and $\mathrm{Fe}$ have increased at WB, consistent with results reported by SanClements (2008) that soil $\mathrm{Al}$ and Fe, and possibly soil $\mathrm{P}$, increased in the upper soil levels at WB.
Mature sugar maple photosynthetic rates were higher in 2002 and 2003 in WB than in EB, but no differences were detected for photosynthetic rates of red spruce and American beech between watersheds (Elvir et al. 2006). They suggested that net photosynthesis of American beech and red spruce in the treated watershed was limited by $\mathrm{Ca}$ and $\mathrm{Mg}$, despite the higher foliar $\mathrm{N}$ concentrations in these species. The findings of Elvir et al. (2006) suggest that higher foliar $\mathrm{N}$ in sugar maple trees in the treated watershed and similar foliar $\mathrm{Ca}$ and $\mathrm{Mg}$ concentrations between trees in the treated and reference watershed gave a physiological advantage to the sugar maple trees in the treated watershed and hence the increased photosynthetic rates. Consistently, Bethers et al. (2009) showed that sugar maple saplings had higher foliar $\mathrm{N}$ but lower $\mathrm{Ca}$ and $\mathrm{Mg}$ in the treated watershed than those in the reference watershed, with sapling photosynthetic rates being similar between treated and reference watersheds. Bethers et al. (2009) also showed that sugar maple saplings had lower photosynthetic capacity, carboxylation capacity, and electron transport in the treated watershed compared to the reference, which suggests that foliar nutrient imbalances may be affecting physiological processes of sugar maple.

Chronic $\mathrm{N}$ and $\mathrm{S}$ deposition has the potential to alter biotic communities and the functioning of terrestrial ecosystems. Evidence of the effects on the vegetation of the $\left(\mathrm{NH}_{4}\right)_{2} \mathrm{SO}_{4}$ treatment applied to the WB included changes in foliar chemistry, radial growth, and physiological processes. The $\mathrm{N}+\mathrm{S}$ addition treatment has altered soil nutrient availability which was reflected in higher foliar N. However, lower foliar $\mathrm{Ca}$ and $\mathrm{Mg}$ concentrations of some tree species in the $\mathrm{N}+\mathrm{S}$ addition watershed may indicate that nutrient imbalances have developed for these species. Those altered nutrient concentrations and higher foliar $\mathrm{Al}$ and $\mathrm{Fe}$ concentrations especially for saplings and ground flora have lowered photosynthetic capacity, carboxylation capacity, and electron transport in sugar maple. The initial increase in sugar maple radial growth appears to be a result of higher $\mathrm{N}$ availability and the ability of those tree species to uptake $\mathrm{Ca}$ and $\mathrm{Mg}$ from lower soil levels. However, as the soil acidification progressed, the stimulus of elevated $\mathrm{N}$ was countered 
by shortages of other nutrients causing reduction in radial growth to pretreatment level after the eighth year of treatment. There has been a decline in foliar base cations between 1993 and 2006. These trends suggest that base cation availability is decreasing through time. Evidence also suggests that forest floor vegetation would be the most affected, as base cation availability is further reduced and $\mathrm{Al}$ and $\mathrm{Fe}$ availability is increased, especially in the upper soil levels.

Acknowledgements This research was supported by grants from the National Science Foundation's Long Term Research on Environmental Biology (DEB0639902), funds from the University of Maine Vice President for Research Maine Economic Improvement Fund (MEIF), and the Maine Agricultural and Forest Experiment Station. Our thanks to Summer Allen for all her help in obtaining the required permissions. Our thanks to two anonymous reviewers for their comments and suggestions.

\section{References}

Aber, J., McDowell, W., Nadelhoffer, K., Magill, A., Berntson, G., Kamakea, M., et al. (1998). Nitrogen saturation in temperate forest ecosystems. Hypotheses revisited. BioScience, 48, 921-934.

Aber, J. D., Melillo, J. M., Nadelhoffer, K. J., Pastor, J., \& Boone, R. D. (1991). Factors controlling nitrogen cycling and nitrogen saturation in northern temperate forest ecosystems. Ecological Applications, 1, 303-315.

Aber, J., Nadelhoffer, K. J., Steudler, P., \& Melillo, J. M. (1989). Nitrogen saturation in northern forest ecosystems. BioScience, 39, 378-386.

Amthor, J. S., Gill, D. S., \& Borman, F. H. (1990). Autumnal leaf conductance and apparent photosynthesis by saplings and sprouts in a recently disturbed northern hardwood forest. Oecologia, 84, 93-98.

Andrews, J. A., \& Siccama, T. G. (1995). Retranslocation of calcium and magnesium at the heartwood-sapwood boundary of Atlantic white cedar. Ecology, 76, 659663.

Bertills, U., \& Näsholm, T. (2000). Effects of nitrogen deposition on forest ecosystems. Report 5067, Swedish Environmental Protection Agency. Retrieved 12 December 2008 from http://www. naturvardsverket.se/bokhandeln/pdf/620-6137-2.

Bethers, S. (2008). The effects of chronically elevated N and $\mathrm{S}$ deposition on the nutrition and physiology of sugar maple saplings at the BBWM. M.Sc. thesis, University of Maine.

Bethers, S., Day, M. E., Wiersma, B., Fernandez, I. J., \& Elvir, J. A. (2009). Effects of chronically elevated nitrogen and sulfur deposition on sugar maple saplings: Nutrition, growth and physiology. Forest Ecology and Management, 258, 895-902.
Bondietti, E. A., Momoshima, N., Shortle, W. C., \& Smith, K. T. (1990). A historical perspective on divalent cation trends in red spruce stem wood and the hypothetical relationship to acid deposition. Canadian Journal of Forest Research, 20, 1850-1858.

Buchmann, N., Oren, R., \& Zimmermann, R. (1995). Response of magnesium-deficient saplings in a young, open stand of Picea abies (L.) Karst. to elevated soil magnesium, nitrogen and carbon. Environmental Pollution, 87(1), 31-43.

Clair, S. B., \& Lynch, J. P. (2005). Base cation stimulation of mycorrhization and photosynthesis of sugar maple on acid soils are coupled by foliar nutrient dynamics. New Phytotogist, 165, 581-590.

Cronan, C. S. (1989). Aluminum toxicity in forests exposed to acidic deposition-The ALBIOS results. Water, Air and Soil Pollution, 48, 181-192.

DeHayes, D. H., Schaberg, P. G., Hawley, G. J., \& Strimbeck, G. R. (1999). Acid rain impacts on calcium and nutrition and forest health. BioScience, 10, 789800.

Dise, N. B., \& Wright, R. F. (1995). Nitrogen leaching from European forests in relation to nitrogen deposition. Forest Ecology and Management, 71, 153-161.

Dreyer, E., Fichter, J., \& Bonneau, M. (1994). Nutrient content and photosynthesis of young yellowing Norway spruce trees (Picea abies L. Karst.) following calcium and magnesium fertilization. Plant and Soil, 160(1), 67-78.

Driscoll, C. T., Lawrence, G. B., Bulger, A. J., Butler, T. J., Cronan, C. S., Eager, C., et al. (2001). Acidic deposition in the northeastern United States: Sources and inputs, ecosystem effects, and management strategies. BioScience, 51, 180-198.

Elvir, J. A., Rustad, L., Fernandez, I., \& Wiersma, G. B. (2005). Temporal response of foliar chemistry to chronic nitrogen and sulfur additions at the Bear Brook Watershed in Maine. Canadian Journal of Forest Research, 35, 1402-1410.

Elvir, J. A., Wiersma, G. B., Greenwood, M., Day, M., \& Fernandez, I. (2006). Effects of enhance nitrogen deposition on foliar chemistry and physiological processes of forest trees at the Bear Brook Watershed in Maine. Forest Ecology and Management, 221, 207214.

Elvir, J. A., Wiersma, G. B., White, A., \& Fernandez, I. (2003). Effects of chronic ammonium sulfate treatment on basal area increment in red spruce and sugar maple at the Bear Brook Watershed in Maine. Canadian Journal of Forest Research, 33, 862869.

Farquhar, G. D., Caemmerer, S. V., \& Berry, J. A. (1980). A biochemical-model of photosynthetic $\mathrm{CO}_{2}$ assimilation in leaves of C-3 species. Planta, 149, 78-90.

Fenn, M. E., Haeuber, R., Tonneses, G. S., Baron, J. S., Grossman-Clarke, S., Hope, D., et al. (2003). Nitrogen emissions, deposition, and monitoring in the Western United States. BioScience, 53, 391-403.

Fernandez, I., Rustad, L., David, M., Nadelhoffer, K., \& Mitchell, M. (1999). Mineral soil and solution responses to experimental $\mathrm{N}$ and $\mathrm{S}$ enrichment at the 
Bear Brook Watershed in Maine (BBWM). Environmental Monitoring and Assessment, 55, 165-185.

Fernandez, I. J., Rustad, L. E., Norton, S. A., Kahl, J. S., \& Cosby, B. J. (2003). Experimental acidification causes soil base-cation depletion at the Bear Brook Watershed in Maine. Soil Science Society of America Journal, 67, 1909-1919.

Glock, W. S. (1937). Principles and methods of treering analysis. Washington: Carnegie Institution of Washington.

Godman, R., Harry, Y., \& Tubbs, C. (1990). Acer saccarrum Sarg. Sugar maple. In R. M. Burns, \& B. H. Honkala (Tech. Coords.), 1990. Silvics of North America (Vol. 2). Hardwoods. Agric. Handb. 654. Washington: U.S. Department of Agriculture, Forest Service.

Horsley, S. B., Long, R. P., Bailey, S. W., Hallett, R. A., \& Hall, T. J. (2000). Factors associated with the decline disease of sugar maple on the Allegheny Plateau. Canadian Journal of Forest Research, 30, 1365-1378.

Houston, D. R., Parker, E. J., \& Lonsdale, D. (1979). Beech bark disease: Patterns of spread and development of the initiating agent Cryptococcus fagisuga. Canadian Journal of Forest Research, 9, 336-343.

Jefts, S., Fernandez, I. J., Rustad, L. E., \& Dail, D. B. (2004). Decadal responses in soil $\mathrm{N}$ dynamics at the Bear Brook Watershed in Maine, USA. Forest Ecology and Management, 189, 189-205.

Johnson, A. H., Anderson, S. B., \& Siccama, T. G. (1994). Acid rain and soils of the Adirondacks. I. Changes in $\mathrm{pH}$ and available calcium. Canadian Journal of Forest Research, 24, 193-198.

Kahl, J., Norton, S., Fernandez, I., Rustad, L., \& Handley, M. (1999). Nitrogen and sulfur input-output budgets in the experimental and reference watershed, Bear Brook Watershed in Maine BBWM. Environmental Monitoring and Assessment, 55, 113-131.

Kenlan, P. H. (2006). The effects of experimental acidification on understory plant communities at the Bear Brook Watershed in Maine. M.S. thesis, University of Maine.

Kolb, T. E., \& McCormick, L. H. (1993). Etiology of sugar maple decline in 4 Pennsylvania stands. Canadian Journal of Forest Research, 23, 2395-2402.

Laing, W., Greer, D., Sun, O., Beets, P., Lowe, A., \& Payn, T. (2000). Physiological impacts of Mg deficiency in Pinus radiata: Growth and photosynthesis. New Phytologist, 146(1), 47-57.

Lehmann, C. M. B., Bowersox, V. C., \& Larson, S. M. (2005). Spatial and temporal trends of precipitation chemistry in the United States, 1985-2002. Environmental Pollution, 135, 347-361.

LI-COR (1998). Using the LI-6400 portable photosynthesis system. Lincoln, Nebraska: LI-COR, Inc.

Lloyd, J. (1999). The $\mathrm{CO}_{2}$ dependence of photosynthesis, plant growth responses to elevated $\mathrm{CO}_{2}$ concentrations and their interaction with soil nutrient status, II. Temperate and boreal forest productivity and the combined effects of increasing $\mathrm{CO}_{2}$ concentrations and increased nitrogen deposition at a global scale. Functional Ecology, 13, 439-459.
Magill, A., Aber, J., Hendricks, J., Bowden, R., Melillo, J., \& Steudler, P. (1997). Biogeochemical responses of forest ecosystems to simulated chronic nitrogen deposition. Ecological Applications, 7(2), 402-415.

Mclaughlin, S. B., Andersen, C. P., Edwards, N. T., Roy, W. K., \& Layton, P. A. (1990). Seasonal patterns of photosynthesis and respiration of red spruce sapling from two elevations in declining southern Appalachian stands North Caroline USA. Canadian Journal of Forest Research, 20(5), 485-495.

Mclaughlin, S. B., \& Wimmer, R. (1999). Calcium physiology and terrestrial ecosystem processes. New Phytologist, 142, 373-417.

McNulty, S. G., Aber, J. D., \& Newman, S. D. (1996). Nitrogen saturation in a high elevation New England spruce-fir stand. Forest Ecology and Management, 84, 109-121.

Mehne-Jakobs, B. (1995). Seasonal development of the photosynthetic performance of Norway spruce (Picea abies (L.) Karst.) under magnesium deficiency. Plant and Soil, (168-169), 255-261.

Mehne-Jakobs, B. (1996). Magnesium deficiency treatment causes reductions in photosynthesis of well-nourished Norway spruce. Trees, 10(5), 293-300.

NADP (2002-2006). National Atmospheric Deposition Program, annual summary. NADP Program Office, Illinois State Water Survey, 2204 Griffith Drive, Champaign, IL 61820. Retrieved 15 November 2008, from http://nadp.sws.uiuc.edu/lib/.

Norton, S., Kahl, J., Fernandez, I., Haines, T., Rustad, L., Nodvin, S., et al. (1999). The Bear Brook Watershed, Maine (BBWM), USA. Environmental Monitoring and Assessment, 55, 7-51.

Oren, R., Werk, K. S., Buchmann, N., \& Zimmermann, R. (1993). Chlorophyll-nutrient relationships identify nutritionally caused decline in Picea abies stands. Canadian Journal of Forest Research, 23(6), 1187-1195.

Peterson, D. L., Arbaugh, M. J., Robinson, L. J., \& Derderian, B. R. (1990). Growth trends of whitebark pine and lodgepole pine in a subalpine Sierra Nevada forest, California, USA. Arctic and Alpine Research, 22, 233-243.

Reynolds, B., Wilson, E. J., \& Emmett, B. A. (1998). Evaluating critical load of nutrient nitrogen and acidity for terrestrial ecosystems using ecosystem-scale experiments (NITREX). Forest Ecology and Management, 101, 81-94.

SanClements, M. (2008). The chemistry of acidic soils in humid, temperate forested watersheds with emphasis on phosphorus, aluminum and iron. Ph.D. thesis, University of Maine, Orono, ME.

SAS Institute (1999). SAS user's guide. Statistic (8th ed.). Cary, NC: SAS Inc.

Schaberg, P. G., Tilley, J. W., Hawley, G. J., DeHayes, D. H., \& Bailey, S. W. (2006). Associations of calcium and aluminum with the growth and health of sugar maple trees in Vermont. Forest Ecology and Management, 223, 159-169.

Shortle, W. C., \& Smith, K. T. (1988). Aluminum-induced calcium deficiency syndrome in declining red spruce. Science, 240, 1017-1018. 
Simonne, A. H., Simonne, E. H., Eitenmiller, R. R., Mills, H. A., \& Cresman, C. P. (1997). Could the Dumas method replace the Kjeldahl digestion for nitrogen and crude protein determinations in foods? Journal of the Science of Food and Agriculture, 73, 39-45.

Spiecker, H., Mielikäinen, K., Köhl, M., \& Skovsgaard, J. P. (1996). Growth trends in European forests. European Forest Institute Research Report No. 5 (p. 372). Berlin: Springer.

Tietema, A., \& Beier, C. (1995). A correlative evaluation of nitrogen cycling in forest ecosystems of the EC projects NITREX and EXMAN. Forest Ecology and Management, 71, 143-151.

Uddameri, V., Norton, S. A., Kahl, J. S., \& Scofield, J. P. (1995). Randomized intervention analysis of the response of the West Bear Brook watershed, Maine to chemical manipulation. Water, Air and Soil Pollution, 79, 193-199.

Vitousek, P. M., Aber, J. D., Howarth, R. W., Likens, G. E., Matson, P. A., Schindler, D. W., et al. (1997). Human alteration of the global nitrogen cycle: Sources and consequences. Ecological Applications, 7, 737750 .

Wang, Z. (1993). Influences of soil type and forest vegetation on the distribution of forest floor nitrogen at the Bear Brook Watershed in Maine (BBWM). M.S. Thesis, University of Maine, Orono, ME.

Wang, Z., \& Fernandez, I. (1999). Soil type and forest vegetation influences on forest floor nitrogen dynamics at the Bear Brook Watershed in Maine (BBWM). Environmental Monitoring and Assessment, 55, 221-234.

Ward, M. (2001). Age-related trends in red spruce needle anatomy and their relationship to declining productivity (pp. 1-110). M.Sc. thesis, The University of Maine, Orono, ME.

Weber, K. L., \& Wiersma, G. B. (1997). Trace element concentrations in mosses collected from a treated experimental forest watershed. Toxicological and Environmental Chemistry, 65, 17-29.

Weikert, R. M., Wedler, M., Lippert, M., Schramel, P., \& Lange, O. L. (1989). Pothosynthetic performance chloroplast pigments and mineral content of various needle age classes of spruce picea-abies with and without the new flush and experimental approach for analyzing forest decline phenomena. Trees, 3(3),161-172.

White, G., Fernandez, I., \& Wiersma, G. (1999). Impact of ammonium sulfate treatment on the foliar chemistry of forest trees at the Bear Brook Watershed in Maine. Environmental Monitoring and Assessment, 55, 235250. 